\title{
Evolution of an extended Ricci flow system
}

\author{
BERNHARD LIST
}

We show that Hamilton's Ricci flow and the static Einstein vacuum equations are closely connected by the following system of geometric evolution equations:

$$
\begin{aligned}
\partial_{t} g & =-2 R c(g)+2 \alpha_{n} d u \otimes d u, \\
\partial_{t} u & =\Delta^{g} u,
\end{aligned}
$$

where $g(t)$ is a Riemannian metric, $u(t)$ a scalar function and $\alpha_{n}$ a constant depending only on the dimension $n \geq 3$. This provides an interesting and useful link from problems in low-dimensional topology and geometry to physical questions in general relativity.

\section{Introduction}

In the last 20 years, the Ricci flow system for a Riemannian metric introduced in [1]

$$
\partial_{t} g=-2 R c(g)
$$

has been used with great success for the construction of canonical metrics on Riemannian manifolds of low dimension. In his first paper on the Ricci flow, Hamilton proved that given an initial metric with positive Ricci curvature in three dimensions, a rescaled solution for $t \rightarrow \infty$ exists and has constant positive curvature. Many further results in direction of a proof of the Poincare conjecture followed. Some of these are collected in [2]. The recent approach of Perelman to prove the full geometrization conjecture added new ideas to Hamilton's program [6,7]. In particular, Perelman showed that the Ricci flow can be interpreted as a gradient flow of an entropy functional and thus that the canonical metrics are stationary points of this entropy in the variational sense.

Our purpose is to develop a corresponding theory for canonical objects with a certain physical interpretation. We prove the existence of an entropy $E$ such that the stationary points are solutions to the static Einstein vacuum 
equations and study an extended parabolic system

$$
\begin{aligned}
& \partial_{t} g=-2 R c(g)+2 \alpha_{n} d u \otimes d u \\
& \partial_{t} u=\Delta^{g} u
\end{aligned}
$$

which is equivalent to the gradient flow of $E$. For applications on noncompact asymptotically flat manifolds, we prove short time existence for the system (1.1) on complete manifolds.

For a closer examination of the solutions, we provide a range of a priori estimates. These include local interior estimates on balls for all derivatives of $R m$ and $u$, but also global supremum bounds and time decay estimates. We obtain a long time existence result from these estimates, saying that solutions continue to exist as long as the curvature $R m$ of $g(t)$ stays bounded.

From the variational structure we obtain a monotonicity formula for the flow, which we use to prove a noncollapsing result. This is crucial to obtain the injectivity radius bounds necessary for a compactness theorem. Using this result, we obtain compactness of a set of solutions satisfying natural bounds.

In addition, we apply the noncollapsing and the a priori estimates to prove that rapidly forming singularities of the flow at finite time can be rescaled to ancient solutions of the Ricci flow. This makes the study of singularity formation (and also the usage of surgery) possible.

A main motivation to study (1.1) stems from its connection to general relativity. An important issue in the numerical evolution of the Einstein equations is the construction of good initial data sets which have to satisfy the so-called constraint equations. In general, this is a hard problem $[8, \S 2.1,9]$. A parabolic system could be used to improve these data sets. Since this should work in particular for static solutions, our system is an interesting candidate for such a smoothing operator. It should be possible to approximate static solutions by solutions to (1.1).

Another application relates to the quasi-local mass definition of Bartnik in [10] and the recent paper of [11]. After adding suitable parabolic boundary conditions, our system could be helpful in the construction of the static minimal mass extension which would provide the minimum in the definition of this mass according to Bartnik's conjecture.

With regard to these applications, we conclude this paper with the study of (1.1) on asymptotically flat manifolds. We consider two classes of asymptotically flat manifolds and prove that for given initial data in one of these classes, the flow preserves the asymptotic structure for $t<\infty$. Moreover, we show that the ADM mass of the initial data is preserved for finite times. 


\section{Preliminaries}

We consider smooth $n$-dimensional complete Riemannian manifolds $\Sigma$ or $M$ where $M$ is always used to specify a closed manifold. For a Riemannian metric $g$ we denote the Levi-Civita connection by $\nabla$ and its curvature tensor by $R m$. The Ricci tensor is denoted by $R c$, the scalar curvature by $R$ and the volume element by $d V$. If we need to make clear to which metric these tensors belong, we write $R m(g)$ and so on. The exterior derivative of a form is denoted by $d$.

Given some coordinate system $\left\{x^{1}, \ldots, x^{n}\right\}$ we abbreviate $\partial_{i}:=\frac{\partial}{\partial x^{i}}$ for the partial derivatives with respect to these coordinates, $\nabla_{i}:=\nabla_{\partial} / \partial_{i}$ for the covariant derivatives associated to $g$, and $\partial_{t}$ for partial differentiation with respect to time. We denote the components of the Hessian $\nabla^{2}$ of some function $u$ by $\nabla_{i} \nabla_{j} u:=\nabla_{i j}^{2} u$ and similar for higher derivatives. The components of the metric $g$ itself are given by $\left\{g_{i j}\right\}$, and the inverse metric $g^{-1}$ on the cotangent bundle $T^{*} \Sigma$ is represented by $\left\{g^{i j}\right\}$. The Laplacian of a function $u$ with respect to $g$ is given by

$$
\Delta^{g} u=g^{i j} \nabla_{i} \nabla_{j} u=g^{i j}\left(\partial_{i} \partial_{j} u-\Gamma_{i j}^{k} \partial_{k} u\right)
$$

Denote the set of smooth Riemannian metrics on $\Sigma$ by $\mathcal{M}(\Sigma)$ and the space of smooth functions by $C^{\infty}(\Sigma)$. The Riemannian metric $g$ induces norms on the tensor algebra. In coordinates it is given for a tensor $B:=\left\{B_{i_{1} \ldots i_{k}}^{j_{1} \ldots j_{l}}\right\}$ by

$$
|B|^{2}:=g^{i_{1} m_{1}} \cdots \cdot g^{i_{k} m_{k}} \cdot g_{j_{1} n_{1}} \cdots g_{j_{l} n_{l}} \cdot B_{i_{1} \cdots i_{k}}^{j_{1} \cdots j_{l}} \cdot B_{m_{1} \cdots m_{k}}^{n_{1} \cdots n_{l}}
$$

where we use the Einstein summation convention, meaning that we sum over a repeated lower and upper index from 1 to $n$. In normal coordinates, the summation can be over two lower indices. The convention is used throughout this paper. We also write $A * B$ for a linear combination of contractions of components of the two tensors $A$ and $B$ when the precise form and number of these terms is irrelevant for the computation. In this notation, factors $g$ and $g^{-1}$ are suppressed.

To motivate our physical interest, we give a short introduction to static vacuum solutions of the Einstein equations. Further material can be found in [12, chapter 2] and [13, chapter 6$]$. A very thorough and detailed discussion is provided in [14].

From a geometrical point of view, a Lorentzian manifold $\left(L^{4}, h\right)$ is said to be static if there exists a 1-parameter group of isometries with timelike orbits and a spacelike hypersurface $\Sigma$ which is orthogonal to these orbits. 
Physically this reflects the fact that the solutions are independent of time, therefore having a time translation and reflection symmetry. Equivalent is the existence of a timelike, hypersurface orthogonal Killing vector field $\xi$.

A vacuum solution to the Einstein equations satisfies $R c(h) \equiv 0$. These solutions describe the gravitational field in a region of spacetime that does not contain any matter.

The combination of these concepts is a static vacuum solution. The most important example in this class is the Schwarzschild solution. It describes the gravitational field in the exterior region around an isolated nonrotating spherically symmetric body like a star or a black hole. In suitably chosen coordinates, a static Einstein vacuum metric has the following simple form:

$$
h=-V^{2}\left(x^{1}, x^{2}, x^{3}\right) d t^{2}+\sum_{i, j=1}^{3} g_{i j}\left(x^{1}, x^{2}, x^{3}\right) d x^{i} d x^{j},
$$

where $\left\{x^{i}\right\}$ are the coordinates on $\Sigma, t$ is the orbit coordinate of $\xi, V:=$ $\sqrt{-h(\xi, \xi)^{2}}$, and $g$ is a Riemannian metric on $\Sigma$. Consequently $h$ is determined by the Lapse function $V$ and the metric $g$ on the spatial slices. The static Einstein vacuum equations in terms of $V$ and $g$ projected onto the hypersurface are given by

$$
\begin{aligned}
R c(g) & =V^{-1} \nabla^{2} V, \\
V^{-1} \Delta^{g} V & =0 .
\end{aligned}
$$

This is shown in $[12,3.4-3.5]$. Vice versa, a pair $(V, g)$ satisfying equation (2.2) gives rise to a uniquely determined static vacuum solution [12, Theorem 2-3.3].

As an example, we apply these ideas to the Schwarzschild solution. The Lorentz metric of the Schwarzschild spacetime is given in spherical spatial coordinates $(t, r, \theta, \phi)$ on $\mathbb{R} \times \mathbb{R}^{3}$ by

$$
h=-\left(1-\frac{2 m}{r}\right) d t^{2}+\left(1-\frac{2 m}{r}\right)^{-1} d r^{2}+r^{2}\left(d \theta^{2}+\sin ^{2} \theta d \phi^{2}\right),
$$

where $m$ is the mass parameter. One calculates that it satisfies (2.2) for $V=\left(1-\frac{2 m}{r}\right)^{1 / 2}$ and $g$ the spatial part of $h$ on the slices $t=$ const.

The system (2.2) can be simplified considerably by removing the second derivatives of $V$ on the right hand side via a conformal transformation. Note that we do not want to restrict ourselves to dimension $n=3$ in the following, but work on manifolds $\Sigma$ of arbitrary dimension $n \geq 3$. 
Lemma 2.1. Define $\tilde{g}:=e^{2 \alpha \psi} \cdot g$ for a function $\psi \in C^{\infty}(\Sigma)$ and a constant $\alpha \in \mathbb{R}$. Let $f \in C^{\infty}(\Sigma)$ be an arbitrary function. Then the following holds:

$$
\begin{aligned}
\tilde{R}_{i j}= & R_{i j}-(n-2) \alpha \nabla_{i} \nabla_{j} \psi+(n-2) \alpha^{2} \partial_{i} \psi \partial_{j} \psi-\alpha \Delta^{g} \psi \cdot g_{i j} \\
& -(n-2) \alpha^{2}|d \psi|_{g}^{2} \cdot g_{i j} \\
\Delta^{\tilde{g}} f= & e^{-2 \alpha \psi}\left(\Delta^{g} f+(n-2) \alpha \cdot g(d \psi, d f)\right) .
\end{aligned}
$$

Defining $\quad u:=\ln V$ and using the conformal transformation $\tilde{g}:=$ $e^{(2 /(n-2)) u} \cdot g$, we get from $(2.2)$ and Lemma 2.1 with $f:=\psi:=u$ and $\alpha:=$ $1 /(n-2)$ :

$$
\begin{aligned}
\tilde{R}_{i j} & =R_{i j}-\nabla_{i} \nabla_{j} u+\frac{1}{n-2} \partial_{i} u \partial_{j} u-\frac{1}{n-2}\left(\Delta^{g} u+|d u|_{g}^{2}\right) \cdot g_{i j} \\
& =\left(\nabla_{i} \nabla_{j} u+\partial_{i} u \partial_{j} u\right)-\nabla_{i} \nabla_{j} u+\frac{1}{n-2} \partial_{i} u \partial_{j} u-\frac{1}{n-2} e^{-u} \Delta^{g} e^{u} \cdot g_{i j} \\
& =\frac{n-1}{n-2} \partial_{i} u \partial_{j} u \\
\Delta^{\tilde{g}} u & =e^{(-2 /(n-2)) u}\left(\Delta^{g} u+\frac{n-2}{n-2}|d u|_{g}^{2}\right)=0 .
\end{aligned}
$$

This is the conformal formulation of the static Einstein vacuum equations for $(\tilde{g}, u)$ :

$$
\begin{aligned}
\tilde{R}_{i j} & =\frac{n-1}{n-2} \partial_{i} u \partial_{j} u \\
\Delta^{\tilde{g}} u & =0
\end{aligned}
$$

which is equivalent to $R c(h)=0$ for the following Lorentz metric on $\mathbb{R} \otimes \mathbb{R}^{n}$ :

$$
h=-e^{(2 /(n-2)) u} d t^{2}+e^{-(2 /(n-2)) u} g_{i j} d x^{i} d x^{j} .
$$

One solution to (2.4) is the conformal equivalent of the Schwarzschild metric (2.3). The spatial metric on the slices $t=$ const is given by $\tilde{g}=d r^{2}+r(r-2 m)$ $\left(d \theta^{2}+\sin ^{2} \theta d \phi^{2}\right)$ and the logarithm of the Lapse function $u:=\ln V=\frac{1}{2}$ $(\ln (r-2 m)-\ln r)$. Other examples are solutions $(g, u)$ on $\mathbb{R} \times M^{n}$ where $M^{n}$ is a Riemannian manifold, $g$ a Ricci-flat metric and $u \equiv$ const.

\section{Flow equations and entropy}

The purpose of this paper is to study the following initial value problem for a Riemannian metric $g(t)$ and a smooth function $u(t)$ together with given 
initial data $(\tilde{g}, \tilde{u}) \in \mathcal{M}(\Sigma) \times C^{\infty}(\Sigma)$ :

$$
\begin{aligned}
& \partial_{t} g=-2 R c(g)+2 \alpha_{n} d u \otimes d u, \\
& \partial_{t} u=\Delta^{g} u
\end{aligned}
$$

where we define $\alpha_{n}:=\frac{n-1}{n-2}$. We also introduce the symmetric tensor field $S y \in \operatorname{Sym}_{2}(\Sigma)$ and its trace $S:=g^{i j} S_{i j}$ :

$$
S_{i j}:=R_{i j}-\alpha_{n} \partial_{i} u \partial_{j} u \quad \text { and } \quad S:=R-\alpha_{n}|d u|^{2} .
$$

An important property of (3.1) is its variational structure. In particular it is equivalent to the gradient flow of the following entropy functional:

Definition 3.1. Let $\Sigma$ be a smooth $n$-dimensional Riemannian manifold for $n \geq 3$. The entropy of a configuration $(g, u, f) \in \mathcal{M}(\Sigma) \times C^{\infty}(\Sigma) \times C^{\infty}(\Sigma)$ is defined as follows:

$$
E_{n}(g, u, f):=\int_{\Sigma}\left(R-\alpha_{n}|d u|^{2}+|d f|^{2}\right) e^{-f} d V .
$$

Following an idea of Perelman in $[6, \S 1]$, we include the diffeomorphisms of $\Sigma$ (with potential function $f(t)$ ) into the entropy and get the variation:

$$
\begin{aligned}
\delta E[g, u, f](v, w, h) \\
=\int_{\Sigma}\left(v_{i j}\left\{-R_{i j}-\nabla_{i} \nabla_{j} f+\alpha_{n} \partial_{i} u \partial_{j} u\right\}\right. \\
\left.\quad+4 \alpha_{n} w\left\{\frac{1}{2} \Delta u-\frac{1}{2}\langle d u, d f\rangle\right\}\right) e^{-f} d V \\
\quad+\int_{\Sigma}\left(\frac{\operatorname{tr} v}{2}-h\right)\left\{2 \Delta f-|d f|^{2}+R-\alpha_{n}|d u|^{2}\right\} e^{-f} d V .
\end{aligned}
$$

Defining $h:=(\operatorname{tr} v) / 2$ (which fixes the volume form $e^{-f} d V$ and therefore couples the variation of $f$ to the variation of $g$ ), we finally arrive at

$$
\begin{aligned}
\delta E[g, u, f](v, w)= & \int_{\Sigma}\left(v_{i j} \cdot\left\{-R_{i j}-\nabla_{i} \nabla_{j} f+\alpha_{n} \partial_{i} u \partial_{j} u\right\}\right. \\
& \left.+4 \alpha_{n} w \cdot\left\{\frac{1}{2} \Delta u-\frac{1}{2}\langle d u, d f\rangle\right\}\right) e^{-f} d V,
\end{aligned}
$$

where $E$ is now a functional of $g$ and $u$ alone since $f$ is determined by $g$ and $u$. We introduce a weighted scalar product on the configuration space 
$H:=\mathcal{M}(\Sigma) \times C^{\infty}(\Sigma):$

$$
\left\langle\left(g_{1}, u_{1}\right),\left(g_{2}, u_{2}\right)\right\rangle_{H}:=\int_{\Sigma}\left(\left\langle g_{1}, g_{2}\right\rangle+4 \alpha_{n}\left\langle u_{1}, u_{2}\right\rangle\right) e^{-f} d V
$$

where $\langle\cdot, \cdot\rangle$ denotes the pointwise Euclidean scalar product. Then the gradient flow for $E_{n}$ after multiplication by 2 is given by (3.2) and the definition of $h$ as follows:

$$
\begin{aligned}
\partial_{t} g_{i j} & :=\left(\operatorname{grad}_{1} E\right)_{i j}=-2 R_{i j}+2 \alpha_{n} \partial_{i} u \partial_{j} u-2 \nabla_{i} \nabla_{j} f \\
\partial_{t} u & :=\left(\operatorname{grad}_{2} E\right)=\Delta u-d u(\nabla f), \\
\partial_{t} f & :=h=\frac{\operatorname{tr} v}{2}=-\Delta f-R+\alpha_{n}|d u|^{2} .
\end{aligned}
$$

The application of the diffeomorphisms generated by $\nabla f(t)$ yields the equivalent system

$$
\begin{aligned}
\partial_{t} g_{i j} & =-2 R_{i j}+2 \alpha_{n} \partial_{i} u \partial_{j} u, \\
\partial_{t} u & =\Delta u, \\
\partial_{t} f & =-\Delta f+|d f|^{2}-R+\alpha_{n}|d u|^{2} .
\end{aligned}
$$

It is crucial that the right hand side of the equation for $g$ only involves first derivatives of $u$. If there is a Hessian term, a transformation in this way is not possible.

If $u \equiv c=$ const and $g(t)$ is an arbitrary solution to Hamilton's Ricci flow, then the pair $(g(t), c)$ solves (3.1). An arbitrary solution $(g, u)$ of the static Einstein vacuum equations (2.4) is automatically a stationary solution of the flow equations. Vice versa the first two equations in (3.4) imply that a stationary solution must satisfy the static Einstein vacuum equations (2.4). Note that the results of this section are in perfect agreement with the work of Perelman in [6] when we set $u \equiv$ const.

We collect the evolution equations we are going to need later on. The complete computations can be found in $[15, \S 2]$.

Lemma 3.2. Let $(g, u)(t)$ be a solution to (3.1). Then the following evolution equations hold:

$$
\begin{aligned}
\partial_{t} R= & \Delta R+2|R c|^{2}+2 \alpha_{n}|\Delta u|^{2}-2 \alpha_{n}\left|\nabla^{2} u\right|^{2}-4 \alpha_{n}\langle R c, d u \otimes d u\rangle, \\
\partial_{t} R_{i j}= & \Delta R_{i j}-2 R_{i p} R_{j p}+2 R_{p i q j}\left(R_{p q}-\alpha_{n} \partial_{p} u \partial_{q} u\right)+2 \alpha_{n} \Delta u \nabla_{i} \nabla_{j} u \\
& -2 \alpha_{n} \nabla_{p} \nabla_{i} u \nabla_{p} \nabla_{j} u,
\end{aligned}
$$




$$
\partial_{t} \Gamma_{i j}^{k}=g^{k l}\left(-\nabla_{i} R_{j l}-\nabla_{j} R_{i l}+\nabla_{l} R_{i j}+2 \alpha_{n} \nabla_{i} \nabla_{j} u \partial_{l} u\right)
$$

$$
\begin{aligned}
\partial_{t}|d u|^{2}= & \Delta|d u|^{2}-2\left|\nabla^{2} u\right|^{2}-2 \alpha_{n}|d u|^{4}, \\
\partial_{t}\left(\nabla_{i} \nabla_{j} u\right)= & \Delta\left(\nabla_{i} \nabla_{j} u\right)+2 R_{i p j q} \nabla_{p} \nabla_{q} u-R_{i p} \nabla_{j} \nabla_{p} u-R_{j p} \nabla_{i} \nabla_{p} u \\
& -2 \alpha_{n}|d u|^{2} \nabla_{i} \nabla_{j} u, \\
\partial_{t} S_{i j}= & \Delta S_{i j}-R_{i p} S_{j p}-R_{j p} S_{i p}-2 R_{p i j q} S_{p q}+2 \alpha_{n} \Delta u \nabla_{i} \nabla_{j} u, \\
\partial_{t} S= & \Delta S+2|S y|^{2}+2 \alpha_{n}|\Delta u|^{2}, \\
\partial_{t} R_{i j k l}= & \Delta R_{i j k l}+2\left(B_{i j k l}-B_{i j l k}-B_{i l j k}+B_{i k j l}\right)-R_{i j p l} R_{k p}+R_{i j k p} R_{l p} \\
& +R_{p j k l} R_{p i}+R_{i p k l} R_{p j}+2 \alpha_{n}\left(\nabla_{i} \nabla_{k} u \nabla_{j} \nabla_{l} u-\nabla_{i} \nabla_{l} u \nabla_{j} \nabla_{k} u\right),
\end{aligned}
$$

$$
\partial_{t}|R m|^{2} \leq \Delta|R m|^{2}-2|\nabla R m|^{2}+C|R m|^{3}+C|R m|\left|\nabla^{2} u\right|^{2}+C|d u|^{2}|R m|^{2},
$$

$$
\partial_{t}\left|\nabla^{2} u\right|^{2} \leq \Delta\left|\nabla^{2} u\right|^{2}-2\left|\nabla^{3} u\right|^{2}+C|R m|\left|\nabla^{2} u\right|^{2}+C|d u|^{2}\left|\nabla^{2} u\right|^{2} .
$$

Here $\left\{B_{i j k l}\right\}$ is the tensor introduced in $[1, \S 7]$ and $C=C(n)$ depends only on $n$.

As an application for (3.6) we prove:

Lemma 3.3. Let $(g, u)(t)$ be a solution to (3.1) on $[0, T) \times M$ for closed $M$ with initial data $(\tilde{g}, \tilde{u})$. Define $c_{0}:=\sup _{M}|d \tilde{u}|_{0}^{2}$, where $|\cdot|$ is with respect to $\tilde{g}$. Assume that the curvature satisfies $|R m|^{2}(t) \leq k_{0}$ for $t \in[0, T)$. Then all metrics $g(t)$ are equivalent and we can estimate

$$
e^{-C T} \tilde{g}(x) \leq g(t, x) \leq e^{C T} \tilde{g}(x) \quad \forall(t, x) \in[0, T) \times M
$$

for a constant $C=C\left(n, k_{0}, c_{0}\right)$ depending only on $n, k_{0}, c_{0}$. In fact $C=$ $2\left(n \sqrt{k_{0}}+\alpha_{n} c_{0}\right)$.

Proof. The proof is straightforward using the maximum principle for $|d u|^{2}$ and Gronwall's inequality [15, Lemma 2.8].

We prove that $E_{n}(t)$ really is an entropy. This lemma holds also on complete manifolds $\Sigma$ as long as the integration by parts can be justified. 
Lemma 3.4. Let $(g, u, f)(t)$ be a solution on $[0, T) \times M$. Then the entropy evolves according to:

$$
\partial_{t} E_{n}(g, u, f)(t)=2 \int_{M}\left(\left|S y+\nabla^{2} f\right|^{2}+\alpha_{n}|\Delta u-d u(\nabla f)|^{2}\right) e^{-f} d V \geq 0 .
$$

In particular the entropy is nondecreasing. Equality holds if and only if $(g, u, f)(t)$ satisfies

$$
S y+\nabla^{2} f=0 \quad \text { and } \quad \Delta u-d u(\nabla f)=0 .
$$

Such a solution is called a gradient soliton.

Proof. Inserting the evolution equations just computed and performing several integrations by parts the result follows from a straightforward calculation; see [15, Lemma 2.13].

\section{Short time existence}

As the Ricci flow, the system (3.1) is weakly parabolic. Therefore we can use DeTurck's method [16] to prove short time existence and uniqueness on closed manifolds. For the complete case we show $[15, \S 3]$ that the proof of Shi [17] can be modified to the situation at hand. This yields the following general existence theorem:

Theorem 4.1. Let $(\Sigma, \tilde{g})$ be a smooth complete noncompact $n$-dimensional Riemannian manifold with $|\tilde{R} m|_{0}^{2} \leq k_{0}$. Let $\tilde{u} \in C^{\infty}(\Sigma)$ satisfy $|\tilde{u}|_{0}^{2}+$ $|\tilde{\nabla} \tilde{u}|_{0}^{2} \leq c_{0}$ and $\left|\tilde{\nabla}^{2} \tilde{u}\right|_{0}^{2} \leq s_{0}$. Then there exists a constant $T=T\left(n, k_{0}, c_{0}\right)>$ $c(n) \cdot 1 /\left(\sqrt{k_{0}}+c_{0}\right)$ such that the initial value problem

$$
\begin{aligned}
& \partial_{t} g=-2 R c+2 \alpha_{n} d u \otimes d u, \\
& \partial_{t} u=\Delta u,
\end{aligned}
$$

with initial values $g(0)=\tilde{g}$ and $u(0)=\tilde{u}$ on $\Sigma$ has a smooth solution $(g, u)(t)$ on $[0, T] \times \Sigma$. Moreover the solution satisfies

$$
C^{-1} \tilde{g} \leq g(t) \leq C \tilde{g} \quad \forall t \in[0, T]
$$

for some constant $C=C\left(n, k_{0}, c_{0}, s_{0}\right)$, and on $[0, T] \times \Sigma$ there is a bound

$$
|R m|^{2}+|u|^{2}+|d u|^{2}+\left|\nabla^{2} u\right|^{2} \leq c=c\left(n, k_{0}, c_{0}, s_{0}\right) .
$$


Proof. We refer to $[15, \S 3]$ for the complete proof and just point out where modifications to the proof for the Ricci flow in [17] have been necessary.

The first step is to prove existence for the modified equivalent strongly parabolic system on compact domains. Using the test function $\varphi$ from $[17$, Lemma 2.2] we see that $g(t)$ can be estimated from below by $\tilde{g}$ since the additional term in the evolution equation for $\varphi$ has a sign. A global bound for $|\tilde{\nabla} u|_{0}^{2}$ [15, Lemma 3.5] together with a continuity argument [15, Lemma 3.6] proves the upper bound for $g(t)$, yielding equivalence of the metrics $g(t)$ and $\tilde{g}[15$, Theorem 3.7]. A careful combination of the evolution equations for $\left|\tilde{\nabla}^{k} g\right|_{0}^{2}$ and $\left|\tilde{\nabla}^{k} u\right|_{0}^{2}$ for all $k \geq 0$ provides the necessary interior a priori estimates [15, Proposition 3.8,3.9]. Here we need the global bound for the gradient of $u$ again to overcome difficulties arising from the appearance of $u$ in the evolution equations of the necessary test functions. In particular, there are differences from the fact that the initial value of these test functions does not vanish anymore.

Collecting these results, the theorem of Arzela-Ascoli provides a sequence of solutions on an exhaustion of $\Sigma$ converging to a limiting global solution of the modified parabolic system [15, Theorem 3.12]. We prove integral estimates [15, Lemma 3.16,3.20] and global supremum estimates [15, Proposition 3.14,3.21] (depending only on the initial bounds on $R m$ and $d u$, whereas the interior estimates depended on the full $C^{\infty}$ norm of $\tilde{g}$ ). This is possible since the estimates for the derivatives of $g$ and $u$ can be combined in the right way. Therefore we can define a smooth pullback of the solution of the modified system to a global solution of the original weakly parabolic system (3.1) together with the stated estimates [15, Theorem 3.22].

Remark 4.2. We do not prove uniqueness of the solution in Theorem 4.1. However, the result in [18] strongly suggests that solutions satisfying the above bounds are unique. In particular [19, Appendix B.3] applies to (3.1) on complete and asymptotically flat manifolds.

\section{A priori estimates and long time existence}

To prove interior estimates, we need a time-dependent scaling function.

Lemma 5.1. Let $R>0$ be a fixed radius and $T>0$ be a fixed time. Let $\varphi:[0, T] \rightarrow \mathbb{R}^{+}$be defined by $\varphi(t):=\frac{R^{2} t}{R^{2}+t}$. It satisfies for all $k \geq 0$ and all $t \in[0, T]:$

$$
\partial_{t} \varphi^{k+1}=(k+1) \varphi^{k}
$$




$$
\varphi \leq R^{2}
$$

We will estimate the solutions on the following union of metric balls at different times.

Definition 5.2. Let $g(t)$ be a time-dependent Riemannian metric on a complete manifold $\Sigma$ with distance function $d_{t}(x, y)$. Let $x_{0} \in \Sigma$ and a radius $R>0$ be given. Then we define

$$
B\left(\tau, x_{0}, R\right):=\bigcup_{t \in[0, \tau]} \bar{B}_{R}^{t}\left(x_{0}\right) \subset[0, \tau] \times \Sigma
$$

as the union of the geodesic balls $\bar{B}_{R}^{t}\left(x_{0}\right):=\left\{(t, x) \in\{t\} \times \Sigma \mid d_{t}\left(x_{0}, x\right) \leq R\right\}$.

We need to estimate the time derivative of the metric distance:

Lemma 5.3. Let $(g, u)(t)$ be a solution to (3.1) on $[0, T] \times \Sigma$. Let $x_{0}, x_{1}$ be two fixed points in $\Sigma$ and $d_{t}\left(x_{0}, x_{1}\right)$ their time-dependent distance. Then we can estimate its time derivative:

$$
-\Lambda d_{t}\left(x_{0}, x_{1}\right) \leq \frac{d}{d t} d_{t}\left(x_{0}, x_{1}\right) \leq \Omega d_{t}\left(x_{0}, x_{1}\right)
$$

whenever $\sup _{[0, T] \times \Sigma}|R c| \leq \Lambda$ and $\sup _{[0, T] \times \Sigma}|S y| \leq \Omega$. The result is still true, if the bounds only hold along all minimizing geodesics between $x_{0}$ and $x_{1}$ at all times $0 \leq t \leq T$.

Proof. Let $\gamma:[0, L] \rightarrow \Sigma$ be a smooth curve parameterized by arc length. We compute the time derivative of the $g(t)$-length of $\gamma$ which exists in the sense of difference quotients:

$$
\partial_{t} L^{t}(\gamma)=\int_{\gamma} \frac{1}{2|\dot{\gamma}|_{t}} \cdot\left(\partial_{t} g_{i j}\right) \dot{\gamma}^{i} \dot{\gamma}^{j} d s=-\int_{\gamma} S y(\dot{\gamma}, \dot{\gamma}) d s
$$

Consider the compact set $\Gamma$ of smooth curves $\gamma$ parameterized by arc length and having at most a finite but large length $L$ :

$$
\Gamma:=\left\{\gamma \mid \gamma:\left[0, L^{0}(\gamma)\right] \rightarrow \Sigma, \gamma(0)=x_{0}, \gamma\left(L^{0}(\gamma)\right)=x_{1}, L^{0}(\gamma) \leq L\right\}
$$

Since $d_{t}\left(x_{0}, x_{1}\right)=\inf _{\gamma \in \Gamma} L^{t}(\gamma)$ by definition, we apply [3, Lemma 3.5] to conclude

$$
-\sup _{\gamma \in \Gamma(t)}\left\{\int_{\gamma} S y(\dot{\gamma}, \dot{\gamma}) d s\right\} \leq \frac{d}{d t}\left(d_{t}\left(x_{0}, x_{1}\right)\right) \leq-\inf _{\gamma \in \Gamma(t)}\left\{\int_{\gamma} S y(\dot{\gamma}, \dot{\gamma}) d s\right\} .
$$


Here $\Gamma(t)$ is the set of minimizing geodesics between $x_{0}$ and $x_{1}$ at time $t$. For a proof see [15, Lemma 6.4]. Now the estimates

$$
\begin{aligned}
-\int_{\gamma} S y(\dot{\gamma}, \dot{\gamma}) d s & =-\int_{\gamma} R c(\dot{\gamma}, \dot{\gamma}) d s+\alpha_{n} \int_{\gamma}|d u(\dot{\gamma})|^{2} d s \geq-\sup _{[0, T] \times \Sigma}|R c| \cdot L^{t}(\gamma) \\
& =-\sup _{[0, T] \times \Sigma}|R c| \cdot d_{t}\left(x_{0}, x_{1}\right) \geq-\Lambda \cdot d_{t}\left(x_{0}, x_{1}\right)
\end{aligned}
$$

and

$$
-\int_{\gamma} S y(\dot{\gamma}, \dot{\gamma}) d s \leq \sup _{[0, T] \times \Sigma}|S y| \cdot d_{t}\left(x_{0}, x_{1}\right) \leq \Lambda \cdot d_{t}\left(x_{0}, x\right)
$$

for all $\gamma \in \Gamma(t)$ imply the desired result.

Lemma 5.3 allows us to compare the distance of points at two different times. In particular, it implies that the sets $B\left(\tau, x_{0}, R\right)$ are compact.

Corollary 5.4. Assume $(g, u)(t)$ is a solution to (3.1) on $[0, T] \times \Sigma$. Let points $x_{0}, x_{1} \in \Sigma$ and times $0 \leq t_{1}, t_{2} \leq T$ be chosen. If $|R c| \leq \Lambda$ and $\mid$ Sy $\mid \leq$ $\Omega$ holds along all minimizing geodesics $\gamma$ connecting $x_{0}$ and $x_{1}$ at times $0 \leq t \leq T$, then the distances can be compared as follows:

$$
e^{-\Lambda\left(t_{2}-t_{1}\right)} d_{t_{1}}\left(x_{0}, x_{1}\right) \leq d_{t_{2}}\left(x_{0}, x_{1}\right) \leq e^{\Omega\left(t_{2}-t_{1}\right)} d_{t_{1}}\left(x_{0}, x_{1}\right)
$$

Proof. We apply Lemma 5.3 and integrate on $\left[t_{1}, t_{2}\right]$. After exponentiation the result follows.

We are in the position now to introduce the cutoff function:

Lemma 5.5. Let $\eta: B\left(T, x_{0}, R\right) \rightarrow \mathbb{R}^{+}$be the cutoff function defined by $\eta(t, x):=\left(R^{2}-\frac{1}{2} d_{t}^{2}\left(x_{0}, x\right)\right)^{2}$. Then for all $\theta \in[0,1)$, it has the properties:

$$
\begin{aligned}
\eta & \leq R^{4} \\
\eta^{-1} & \leq(1-\theta)^{-2} R^{-4} \quad \text { on } B\left(T, \theta R, x_{0}\right), \\
|\nabla \eta|^{2} & \leq 4 R^{2} \cdot \eta .
\end{aligned}
$$

Whenever $\sup _{B\left(T, x_{0}, R\right)}|R c| \cdot R^{2} \leq \tilde{C}$ holds, there is the additional estimate

$$
\left(\partial_{t}-\Delta\right) \eta \leq C(n) \tilde{C} \cdot R^{2}
$$

for a scaling invariant constant $\tilde{C}$ and $C(n)$ depending only on $n$. 
Proof. Equations (5.3) and (5.4) are immediate. Equation (5.5) follows from $|\nabla d|=1$. The Laplacian Comparison Theorem [20, Corollary 1.2] and Lemma 5.3 yield an estimate for $\left(\Delta-\partial_{t}\right) d_{t}$. See $[15$, Lemma 6.8] for computational details.

The evolution equations for $u$ and $|d u|^{2}$ give us good control on the behavior of the logarithm of the Lapse function. We start on closed $M$ :

Lemma 5.6. Let $(g, u)(t)$ be a solution on $[0, T) \times M$ with initial data $(\tilde{g}, \tilde{u})$. Then for all $t \in(0, T]$ the following a priori estimates hold:

$$
\begin{aligned}
& \sup _{x \in M}|d u|^{2}(t, x) \leq \max _{x \in M}|d \tilde{u}|_{0}^{2}(x) \\
& \sup _{x \in M}|d u|^{2}(t, x) \leq \frac{1}{2 \alpha_{n}} t^{-1} .
\end{aligned}
$$

Proof. The compact maximum principle applied to (3.6) proves (5.7). A closer look reveals

$$
\begin{aligned}
\partial_{t}\left(t \cdot|d u|^{2}\right) \leq & 1 \cdot|d u|^{2}+t\left(\Delta u-2\left|\nabla^{2} u\right|^{2}-2 \alpha_{n}|d u|^{4}\right) \leq \Delta\left(t \cdot|d u|^{2}\right) \\
& +t^{-1}\left(t|d u|^{2}-2 \alpha_{n} t^{2}|d u|^{4}\right)
\end{aligned}
$$

such that we get at the first point $\left(t^{*}, x^{*}\right) \in[0, \tau] \times M$, with $\tau<T$ arbitrary, where $f:=t \cdot|d u|^{2}$ attains its maximum:

$$
0 \leq t^{-1}\left(f-2 \alpha_{n} f^{2}\right)
$$

This implies for $t>0$

$$
f\left(1-2 \alpha_{n} f\right) \geq 0
$$

which forces $f \leq \frac{1}{2 \alpha_{n}}$ on $(0, T) \times M$, implying $|d u|^{2} \leq \frac{1}{2 \alpha_{n}} t^{-1}$ independent of the initial data. In addition, this yields a uniform bound on $\left[\frac{T}{2}, T\right)$ and therefore the claim for $t=T$.

Using (3.6) and (3.8), we can control the Hessian of $u$ on closed manifolds:

Proposition 5.7. Let $(g, u)(t)$ be a solution to (3.1) for $t \in[0, T)$ on a closed manifold $M$ with initial data $(\tilde{g}, \tilde{u})$. Define $c_{0}:=\max _{M}|d \tilde{u}|_{0}^{2}$ and $s_{0}:=\max _{M}\left|\tilde{\nabla}^{2} \tilde{u}\right|_{0}^{2}$. If $|R m|^{2} \leq k_{0}$ holds on $[0, T) \times M$, then there is a constant $c=c(n)$ depending only on $n$ such that $\left(\nabla^{2} u\right)(t)$ satisfies:

$$
\left.\left|\nabla^{2} u\right|^{2}(t) \leq s_{0}+c(n)\left(\sqrt{k_{0}}+c_{0}\right)\right) \cdot c_{0} \quad \forall t \in[0, T) .
$$


Proof. Equation (5.7) shows that $|d u|^{2}(t, x)$ is bounded on $[0, T] \times M$ by its initial value $c_{0}$. Combining the evolution equations for $|d u|^{2}$ and $\left|\nabla^{2} u\right|^{2}$ from (3.8), we find

$$
\left(\partial_{t}-\Delta\right)\left(\left|\nabla^{2} u\right|^{2}+\lambda|d u|^{2}\right) \leq\left(C_{1}|R m|+C_{2}|d u|^{2}-2 \lambda\right)\left|\nabla^{2} u\right|^{2}
$$

for constants $C_{1}, C_{2}$ depending only on $n$. Choose the constant $2 \lambda$ bigger than $C_{1}|R m|+C_{2}|d u|^{2} \leq C_{1} \cdot \sqrt{k_{0}}+C_{2} \cdot c_{0}$ using the curvature bound and the bound for $|d u|$. This yields

$$
\left|\nabla^{2} u\right|^{2}(t) \leq\left|\nabla^{2} u\right|^{2}(0)+\lambda|d u|^{2}(0) \leq s_{0}+\lambda\left(n, k_{0}, c_{0}\right) \cdot c_{0}
$$

for all $t \in[0, T)$ by the maximum principle since the right hand side of (5.9) is then negative.

To prove a priori estimates for solutions of (3.1), it is useful to collect the component functions of $R m(g)$ and $\nabla^{2} u$ in a vector-valued function $\Phi$ as follows:

$$
\Phi:=\left(R_{i j k l}, \nabla_{p} \nabla_{q} u\right), \quad i, j, k, l, p, q=1, \ldots, n .
$$

We estimate $\Phi$ pointwise using the Euclidean vector norm in $p \in \Sigma$

$$
|\Phi|_{p}^{2}:=|R m|_{p}^{2}+\left|\nabla^{2} u\right|_{p}^{2}
$$

which is the representation of the norm (2.1) in normal coordinates at $p$. This combination of tensors is natural considering their scaling properties. We extend the definition accordingly to higher derivatives and prove:

Lemma 5.8. Let $(g, u)(t)$ be a solution to (3.1). Then for all $k \geq 0$ there exists a constant $C=C(n, k)$ such that we have the inequality

$$
\begin{aligned}
\partial_{t}\left|\nabla^{k} \Phi\right|^{2} \leq & \Delta\left|\nabla^{k} \Phi\right|^{2}-2\left|\nabla^{k+1} \Phi\right|^{2} \\
& +C\left\{\sum_{\alpha+\beta=k}\left|\nabla^{\alpha} \Phi\right|\left|\nabla^{\beta} \Phi\right|\left|\nabla^{k} \Phi\right|+\sum_{\alpha+\beta=k-1}|d u|\left|\nabla^{\alpha} \Phi \| \nabla^{\beta} \Phi\right|\left|\nabla^{k} \Phi\right|\right. \\
& \left.+\sum_{\alpha+\beta+\gamma=k-2}\left|\nabla^{\alpha} \Phi\right|\left|\nabla^{\beta} \Phi\right|\left|\nabla^{\gamma} \Phi\right|\left|\nabla^{k} \Phi\right|+|d u|^{2}\left|\nabla^{k} \Phi\right|^{2}\right\} .
\end{aligned}
$$

Proof. The evolution equations for $\nabla^{k} R m$ and $\nabla^{k+2} u$ are proven by induction on $k$. Since the structure is similar, they can be combined to an equation 
for $\nabla^{k} \Phi$. An application of the Cauchy-Schwarz inequality yields the result. The complete calculation is done in $[15, \S 2.7]$.

Since we can estimate $|R m|^{2}+\left|\nabla^{2} u\right|^{2}$ for solutions of (3.1) on complete $\Sigma$, it is possible to prove a priori bounds for the logarithm of the Lapse function $u(t)$ also on complete, noncompact manifolds. To this end, we need a maximum principle for these manifolds:

Theorem 5.9. Let $\Sigma$ be a complete Riemannian manifold and $(g, u)(t)$ be the solution of (3.1) on $[0, T] \times \Sigma$ constructed in Theorem 4.1 with $T<\infty$. Let $f$ be a smooth function on $[0, T] \times \Sigma$. Assume that there is a vector field $a \in \mathcal{X}([0, T] \times \Sigma)$, and a function $b \in C^{\infty}([0, T] \times \Sigma)$ satisfying $\sup _{[0, T] \times \Sigma}$ $(|a|+|b|) \leq \alpha$, and that

$$
\begin{aligned}
\left(\partial_{t}-\Delta\right) f & \leq a \cdot \nabla f+b f \\
f(0) & \leq 0 \quad \text { on } \Sigma, \\
|\nabla f|^{2} & \leq \beta \quad \text { on }[0, T] \times \Sigma
\end{aligned}
$$

holds for some numbers $\alpha, \beta<\infty$. Then $f \leq 0$ holds on $[0, T] \times \Sigma$.

Proof. This is a specialization of the quite general maximum principle [21, Theorem 4.3]. Using the knowledge on solutions of (3.1) this version is proven in [15, Theorem 6.10].

This theorem not only yields the time decay estimate for $d u$ but also a supremum bound on $u$.

Lemma 5.10. Let $(g, u)(t)$ be the solution from Theorem 4.1 on $[0, T) \times \Sigma$ with initial value $(\tilde{g}, \tilde{u})$. Then the following bounds hold for all $t>0$ as long as the solution exists:

$$
\begin{gathered}
\inf _{x \in \Sigma} \tilde{u}(x) \leq u(t, x) \leq \sup _{x \in \Sigma} \tilde{u}(x) \\
\sup _{x \in \Sigma}|d u|^{2}(t, x) \leq B \cdot t^{-1} .
\end{gathered}
$$

where $B:=\sup _{x \in \Sigma}|\tilde{u}|_{0}^{2}(x)$.

Proof. We apply Theorem 5.9 to $u_{1}(t, x):=u(t, x)-\sup _{x \in \Sigma} \tilde{u}(x)$ and $u_{2}(t, x):=\inf _{x \in \Sigma} \tilde{u}(x)-u(t, x)$. Using the bound $|d u|^{2} \leq C=C\left(n, k_{0}, c_{0}, s_{0}\right)$ 
from Theorem 4.1 the first claim follows. For the second, we use the test function $f:=t|d u|^{2}+|u|^{2}-B$. Theorem 5.9 implies that

$$
0 \geq f(t)=t|d u|^{2}+|u|^{2}-B \quad \Rightarrow \quad t|d u|^{2} \leq B-|u|^{2} \leq B
$$

for all $t>0$ in view of (3.6) and the bound on $|u|^{2}+|d u|^{2}+\left|\nabla^{2} u\right|^{2}$ from Theorem 4.1 .

In addition to the global estimates above we also prove a local bound for $|d u|^{2}$. The technique is adapted from $[21, \S 3]$ and goes back to [17]. We will use the same ideas for the more complicated estimates to follow.

Lemma 5.11. Let $(g, u)(t)$ be a solution to $(3.1)$ on $[0, T) \times \Sigma$. Fix $x_{0} \in \Sigma$ and a radius $R>0$. If there is an estimate

$$
\sup _{B\left(T, x_{0}, R\right)} R^{2}|R c| \leq \tilde{C}
$$

then for all $\theta \in[0,1)$ and all $t \in(0, T]$ there is a constant $C(n)$, depending only on $n$, such that

$$
\sup _{x \in B_{\theta R}^{t}\left(x_{0}\right)}|d u|^{2}(t, x) \leq C(n)(1-\theta)^{-2} \tilde{C}\left(\frac{1}{R^{2}}+\frac{1}{t}\right) .
$$

Proof. We define $f:=\varphi \cdot|d u|^{2}$. A calculation using (3.6) and (5.1) shows

$$
\left(\partial_{t}-\Delta\right) f \leq \varphi^{-1}\left(f-2 \alpha_{n} f^{2}\right)
$$

We multiply by the cut-off function $\eta$ defined in Lemma 5.5 and calculate on $B\left(T, x_{0}, R\right)$ :

$$
\left(\partial_{t}-\Delta\right)(f \eta) \leq \varphi^{-1}\left(f-2 \alpha_{n} f^{2}\right) \cdot \eta-2 \nabla \eta \nabla f+f \cdot C(n) \tilde{C} R^{2}
$$

from (5.6) using the curvature bound. Rewriting the second term and applying (5.5)

$$
-2 \nabla \eta \nabla f=-2 \eta^{-1} \nabla \eta \nabla(\eta f)+2 \eta^{-1}|\nabla \eta|^{2} f \leq-2 \eta^{-1} \nabla \eta \nabla(\eta f)+8 R^{2} f,
$$

we find

$$
\begin{aligned}
\left(\partial_{t}-\Delta\right)(f \eta) \leq & -2 \alpha_{n} \varphi^{-1} f^{2} \eta+\varphi^{-1} f \eta-2 \eta^{-1} \nabla \eta \nabla(f \eta) \\
& +C(n) \tilde{C} R^{2} \cdot f+8 R^{2} \cdot f .
\end{aligned}
$$


Fix $\tau \in[0, T)$. Since $\varphi(0)=0, \eta_{\mid \partial B_{R}^{t}\left(x_{0}\right)}=0$ for all $t \in[0, \tau]$ and $f \eta \geq 0$, the first maximum point $\left(t^{*}, x^{*}\right)$ of $f \eta$ in the compact set $B\left(\tau, x_{0}, R\right)$ must be an interior point. Consequently,

$$
\partial_{t}(f \eta) \geq 0, \quad \Delta(f \eta) \leq 0, \quad \nabla(f \eta)=0,
$$

holds at $\left(t^{*}, x^{*}\right)$ and we obtain, assuming $\tilde{C} \geq 1$ :

$$
2 \alpha_{n} \varphi^{-1} f^{2} \eta \leq \varphi^{-1} f \eta+C(n) \tilde{C} R^{2} \cdot f .
$$

Using (5.3) on the right hand side and multiplying by $\varphi \eta$, we find

$$
2 \alpha_{n} f^{2} \eta^{2} \leq f \eta \cdot R^{4}+C(n) \tilde{C} R^{2} \varphi \cdot f \eta \leq\left(2 \alpha_{n}-1\right) f^{2} \eta^{2}+C(n) \tilde{C}^{2} R^{8}
$$

due to Young's inequality and (5.2). Since $\left(t^{*}, x^{*}\right)$ was a maximum point, we get

$$
\sup _{B\left(\tau, x_{0}, R\right)} f \eta \leq C(n) \tilde{C} R^{4} .
$$

The estimate (5.4) for $\eta^{-1}$ together with (5.13) implies that for any $\theta \in[0,1)$

$$
\sup _{x \in B_{\theta R}^{t}\left(x_{0}\right)}|d u|^{2}(t, x) \leq C(n)(1-\theta)^{-2} \tilde{C} \varphi^{-1}
$$

holds, proving the lemma for all $0<t<T$. Since the estimate is uniform on $[T / 2, T)$, it also holds for $t=T$, completing the proof of Lemma 5.11.

We estimate the derivatives of $\Phi$ where $\Phi$ is defined as in (5.10), giving an explicit dependence of the result on the initial curvature bound. This constitutes a regularity theory for the solutions of (3.1) in the sense that solutions with bounded curvature are always smooth.

Theorem 5.12. Let $(\Sigma, \tilde{g})$ be complete. Let $(g, u)(t)$ be a solution to (3.1) on $[0, T) \times \Sigma$ satisfying

$$
\sup _{B\left(T, x_{0}, R\right)} R^{4}|R m|^{2} \leq \tilde{C}^{2}
$$

for some radius $R>0$ and some point $x_{0} \in \Sigma$. Then the derivatives of $\Phi$ satisfy for all $m \geq 0$ and for all $t \in(0, T]$ the estimates

$$
\sup _{x \in B_{R / 2}^{t}\left(x_{0}\right)}\left|\nabla^{m} \Phi\right|^{2}(t, x) \leq C(n, m) \tilde{C}^{m+2}\left(\frac{1}{R^{2}}+\frac{1}{t}\right)^{m+2},
$$

where $C=C(n, m)$ is a constant depending only on $n$ and $m$. 
Proof. The proof is an induction argument, using similar techniques as in the proof of Lemma 5.11. The curvature bound (5.14) together with Lemma 5.11 provides the estimate

$$
\sup _{B\left(T, x_{0}, \theta_{-1} R\right)} \varphi|d u|^{2} \leq C \tilde{C}
$$

for $\theta_{-1}:=\frac{5}{6}$. Similarly, we estimate $\nabla^{2} u$ using $\varphi|R m| \leq R^{2}|R m|$ in combination with (5.14). See [15, Proposition 6.14] for details. Setting $\theta_{0}:=\frac{3}{4}$, we therefore find that

$$
\begin{aligned}
\sup _{B\left(T, x_{0}, \theta_{0} R\right)} \varphi^{2}|\Phi|^{2} & =\sup _{B\left(T, x_{0}, \theta_{0} R\right)} \varphi^{2}\left(|R m|^{2}+\left|\nabla^{2} u\right|^{2}\right) \\
& \leq \tilde{C}^{2}\left(1+C\left(1-\theta_{0}\right)^{-2}\right) \leq C \tilde{C}^{2}
\end{aligned}
$$

holds, where $C$ depends only on $n$. This proves the theorem in the case where $m=0$. Denote in the following by $C$ a constant depending only on $n$ and $m$ which can change its value from line to line. In the induction step, we assume that

$$
\sup _{x \in B_{\theta_{s}}^{t}\left(x_{0}\right)} \varphi^{s+2}\left|\nabla^{s} \Phi\right|^{2}(t, x) \leq C\left(1-\theta_{s}\right)^{-2} \tilde{C}^{s+2} \leq C \tilde{C}^{s+2}
$$

holds for all $t \in(0, T]$ and all $0 \leq s \leq m$. The choice of $\theta_{s}:=\frac{1}{2}+\frac{1}{s+4}$ guarantees $\frac{1}{2}<\theta_{s}<1$ for all $s$ and $\theta_{i}>\theta_{j}$ for all $i<j$. We assume without loss of generality that $\tilde{C} \geq 1$ in the following. To prove the estimate for $s=m+1$, we define a test function

$$
f(t, x):=\varphi^{m+3}(t)\left|\nabla^{m+1} \Phi\right|^{2}(t, x)\left(\lambda+\varphi^{m+2}(t)\left|\nabla^{m} \Phi\right|^{2}(t, x)\right),
$$

where $\lambda$ is constant and will be chosen later. The evolution of $f$ is given by

$$
\begin{aligned}
\left(\partial_{t}-\Delta\right) f= & \left(\partial_{t}-\Delta\right)\left(\varphi^{m+3}\left|\nabla^{m+1} \Phi\right|^{2}\right) \cdot\left(\lambda+\varphi^{m+2}\left|\nabla^{m} \Phi\right|^{2}\right) \\
& +\varphi^{m+3}\left|\nabla^{m+1} \Phi\right|^{2} \cdot\left(\partial_{t}-\Delta\right)\left(\varphi^{m+2}\left|\nabla^{m} \Phi\right|^{2}\right) \\
& -2 \varphi^{m+3} \nabla\left|\nabla^{m+1} \Phi\right|^{2} \varphi^{m+2} \nabla\left|\nabla^{m} \Phi\right|^{2} .
\end{aligned}
$$

We estimate the individual terms on $B_{\theta_{m} R}^{t}\left(x_{0}\right)$, using the estimate for the derivatives of $\Phi$ from Lemma 5.8 and the evolution equation for $\varphi$ (5.1).

Pairing the correct powers of $\varphi$ with the derivatives of $\Phi$ such that the product is scaling invariant and applying the induction hypotheses (5.15) 
and Young's inequality, we find for the first term:

$$
\begin{aligned}
\left(\partial_{t}-\Delta\right) \varphi^{m+3}\left|\nabla^{m+1} \Phi\right|^{2} \leq & -2 \varphi^{m+3}\left|\nabla^{m+2} \Phi\right|^{2}+C \tilde{C} \varphi^{-1} \cdot \varphi^{m+3}\left|\nabla^{m+1} \Phi\right|^{2} \\
& +C \tilde{C}^{m+4} \varphi^{-1} .
\end{aligned}
$$

The second term in (5.16) can be estimated analogously:

$$
\begin{aligned}
& \left(\partial_{t}-\Delta\right)\left(\lambda+\varphi^{m+2}\left|\nabla^{m} \Phi\right|^{2}\right) \cdot \varphi^{m+3}\left|\nabla^{m+1} \Phi\right|^{2} \\
& \quad \leq-\frac{3}{2} \varphi^{2 m+5}\left|\nabla^{m+1} \Phi\right|^{4}+C \varphi^{-1} \tilde{C} f+C \varphi^{-1} \tilde{C}^{2(m+3)} .
\end{aligned}
$$

The cross term in (5.16) is controlled using the Katos inequality as follows:

$$
\begin{aligned}
- & 2 \varphi^{m+3} \nabla\left|\nabla^{m+1} \Phi\right|^{2} \varphi^{m+2} \nabla\left|\nabla^{m} \Phi\right|^{2} \\
\leq & \varphi^{m+3}\left(2\left|\nabla^{m+2} \Phi\right|\left(\lambda+\varphi^{m+2}\left|\nabla^{m} \Phi\right|^{2}\right)^{1 / 2}\right) \\
& \cdot\left(\left(\lambda+\varphi^{m+2}\left|\nabla^{m} \Phi\right|^{2}\right)^{-1 / 2} \cdot 4 \varphi^{m+2}\left|\nabla^{m} \Phi\right|\left|\nabla^{m+1} \Phi\right|^{2}\right) \\
\leq & 2 \varphi^{m+3}\left|\nabla^{m+2} \Phi\right|^{2}\left(\lambda+\varphi^{m+2}\left|\nabla^{m} \Phi\right|^{2}\right) \\
& +\frac{8 \varphi^{m+2}\left|\nabla^{m} \Phi\right|^{2}}{\lambda+\varphi^{m+2}\left|\nabla^{m} \Phi\right|^{2}} \cdot \varphi^{2 m+5}\left|\nabla^{m+1} \Phi\right|^{4} .
\end{aligned}
$$

Altogether the evolution (5.16) of $f$ comes down to

$$
\begin{aligned}
\left(\partial_{t}-\Delta\right) f \leq & \varphi^{2 m+5}\left|\nabla^{m+1} \Phi\right|^{4}\left(\frac{8 \varphi^{m+2}\left|\nabla^{m} \Phi\right|^{2}}{\lambda+\varphi^{m+2}\left|\nabla^{m} \Phi\right|^{2}}-\frac{3}{2}\right)+C \tilde{C} \varphi^{-1} f \\
& +C \varphi^{-1} \tilde{C}^{m+4}\left(\lambda+\varphi^{m+2}\left|\nabla^{m} \Phi\right|^{2}\right)+C \varphi^{-1} \tilde{C}^{2(m+3)} .
\end{aligned}
$$

We choose $\lambda:=7 \tilde{C}^{m+2} \geq 7 \varphi^{m+2}\left|\nabla^{m} \Psi\right|^{2} \geq 1$ and compute

$$
\frac{8 \varphi^{m+2}\left|\nabla^{m+2} \Phi\right|^{2}}{\lambda+\varphi^{m+2}\left|\nabla^{m} \Phi\right|^{2}}-\frac{3}{2} \leq-\frac{1}{2} \quad \text { and } \quad\left(\lambda+\varphi^{m+2}\left|\nabla^{m} \Phi\right|^{2}\right) \leq 8 \tilde{C}^{m+2} .
$$

Then the first term can be completed to $f^{2}$ and we conclude again applying Young's inequality:

$$
\left(\partial_{t}-\Delta\right) f \leq-\frac{1}{256} \tilde{C}^{-2(m+2)} \varphi^{-1} f^{2}+C \tilde{C}^{2(m+3)} \varphi^{-1} .
$$

To localize this estimate, we multiply $f$ by $\eta$ from Lemma 5.5 and get on the ball $B_{\theta_{m} R}^{t}\left(x_{0}\right)$ :

$$
\begin{aligned}
\left(\partial_{t}-\Delta\right)(f \eta) \leq & -\frac{1}{256} \tilde{C}^{-2(m+2)} \varphi^{-1} f^{2} \eta+C \tilde{C}^{2(m+3)} \varphi^{-1} \eta-2 \eta^{-1} \nabla \eta \nabla(\eta f) \\
& +C(1+\tilde{C}) R^{2} \cdot f
\end{aligned}
$$


where we used the evolution equation (5.6) for $\eta$. Fix $\tau \in[0, T)$. At the first maximum point $\left(t^{*}, x^{*}\right)$ in $B\left(\tau, x_{0}, \theta_{m}\right)$, we get (similarly to the proof of Lemma 5.11) with $\tilde{C} \geq 1$ that

$$
0 \leq-\frac{1}{256} \tilde{C}^{-2(m+2)} \varphi^{-1} f^{2} \eta+C \tilde{C}^{2(m+3)} \varphi^{-1} \eta+C \tilde{C} R^{2} f .
$$

This can be simplified using $\eta^{-1} \leq\left(1-\theta_{m+1}\right)^{-2} R^{-4} \leq C R^{-4}$ on $B_{\theta_{m+1}}^{t}\left(x_{0}\right)$ from (5.4) to

$$
\begin{aligned}
\left|\nabla^{m+1} \Phi\right|^{2} & \leq C \tilde{C}^{2 m+5}\left(\lambda+\varphi^{m+2}\left|\nabla^{m} \Phi\right|^{2}\right)^{-1} \varphi^{-(m+3)} \\
& \leq C \tilde{C}^{2 m+5}\left(7 \tilde{C}^{m+2}\right)^{-1} \varphi^{-(m+3)}
\end{aligned}
$$

for all $(t, x) \in B\left(\tau, x_{0}, \theta_{m+1} R\right)$ because $\left(t^{*}, x^{*}\right)$ was maximal in $B\left(\tau, x_{0}\right.$, $\left.\theta_{m} R\right)$. Since $\tau \in[0, T)$ is arbitrary, this estimate is uniform on $\left[\frac{T}{2}, T\right)$ and therefore also valid for $t=T$. This proves the induction step, and since $\theta_{m}>\frac{1}{2}$ for all $m \geq 0$, it also proves the theorem.

Other versions of the theorem are given in $[15, \S 6.3]$. We use the interior estimates to prove a characterization of long time existence for solutions of (3.1). To this end, we first deduce some general properties for solutions on compact manifolds.

Proposition 5.13. Let $(g, u)(t)$ be a solution to $(3.1)$ on $[0, T) \times M$. Then there is a constant $c=c(n)$ such that we get for all $0 \leq t \leq \min \{T, c / K(0)\}$ where $K(t):=\sup _{x \in M}|R m|(t, x)$ :

$$
K(t) \leq 2 K(0)
$$

Proof. We examine the evolution equation (3.7):

$$
\partial_{t}|R m|^{2} \leq \Delta|R m|^{2}+C|R m|^{3}+C|R m|\left|\nabla^{2} u\right|^{2}+C|d u|^{2}|R m|^{2} .
$$

$K(t)$ is Lipschitz continuous and its derivative (in the sense of difference quotients) satisfies

$$
\frac{d}{d t} K(t)^{2} \leq C K(t)^{3}
$$

since we have $|d u|^{2}(t) \leq C K(t)$ from Lemma 5.11 and $\left|\nabla^{2} u\right|^{2}(t) \leq C K^{2}(t)$ from Theorem 5.12 where $C$ depends only on $n$. Solving the associated 
ordinary differential equation, we find

$$
K(t) \leq\left(\frac{1}{K(0)}-\frac{C}{2} t\right)^{-1}
$$

for $t<\frac{2}{C K(0)}$. Taking $c=1 / C$ yields the claim.

Corollary 5.14. Let $(g, u)(t)$ be a solution to $(3.1)$ on $[0, T) \times M$ where $T$ is the maximal time of existence. Define $k_{0}:=\max _{x \in M}|R m|^{2}(0, x)$. Then there is $c=c(n)$ such that $T>c / \sqrt{k_{0}}$.

Proof. From Proposition 5.13 we know that the solution has bounded curvature on $\left[0, c / \sqrt{k_{0}}\right]$. This implies the smoothness of $(g, u)(t)$ up to that time using Theorem 5.12.

The necessary and sufficient condition for long time existence of solutions on a closed manifold $M$ is given as follows:

Theorem 5.15. Let $(g, u)(t)$ be a solution to $(3.1)$ on $[0, T) \times M$ for closed $M$ with initial data $(\tilde{g}, \tilde{u})$. Assume that $T<\infty$ is maximally chosen such that the solution cannot be extended beyond $T$. Then the curvature of $g(t)$ has to become unbounded for $t \rightarrow T$ in the sense that

$$
\lim _{t \nearrow T}\left[\sup _{x \in M}|R m|^{2}(t, x)\right]=\infty
$$

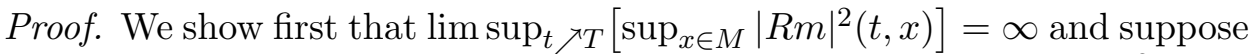
to the contrary that the curvature stays bounded on $[0, T]$, say $|R m|^{2} \leq k_{0}$. Inequality (5.7) implies $|R m|+|d u|^{2} \leq \tilde{C}$ on $[0, T] \times M$. In addition, we know from Theorem 5.12 that the solution is smooth on $[0, T]$. In particular, we can take a smooth limit for $t \rightarrow T$. Taking $(g, u)(T)$ as initial data, the short time existence result provides a solution on a time interval $[T, T+\delta)$. This solution extends the original one smoothly beyond $T$ since the bounds in Theorem 5.12 imply bounds also for all time derivatives of $g(T)$. This contradicts the choice of $T$. The limits superior can be replaced by a proper limit similar to [22, Theorem 6.45] using Proposition 5.13.

\section{The monotonicity formula}

Since the entropy $E$ is not sufficient for all our purposes, we replace it by a scaling invariant integral. To this end, we introduce an explicit scale 
parameter $\tau$ as it is done in [6, §3]. One can think of $\tau$ as time measured backwards from some fixed time. We define the entropy $W$ as

$$
W(g, u, f, \tau):=\int_{M}\left[\tau\left(S+|d f|^{2}\right)+f-n\right](4 \pi \tau)^{-n / 2} e^{-f} d V
$$

for a positive real number $\tau$. It is straightforward to see that $W$ is invariant under diffeomorphisms and scaling invariant for all constants $\alpha>0$ in the following sense:

$$
W(\alpha g, u, f, \alpha \tau)=W(g, u, f, \tau) .
$$

A calculation as before yields the variation

$$
\begin{aligned}
\delta W[g, u, f, \tau](v, w, h, \sigma) & \\
= & \int_{M} v_{i j} \cdot\left\{-\tau S_{i j}-\tau \nabla_{i} \nabla_{j} f\right\}+4 \alpha_{n} w \cdot\left\{\frac{\tau}{2} \Delta u-\frac{\tau}{2}\langle d u, d f\rangle\right\} d m \\
& +\int_{M}\left[\left(\frac{\operatorname{tr} v}{2}-h\right) \cdot\left\{2 \tau \Delta f-2 \tau|d f|^{2}\right\}+h+\sigma \cdot\left\{S+|d f|^{2}\right\}\right] d m \\
& +\int_{M}\left(\frac{\operatorname{tr} v}{2}-h-\frac{n \sigma}{2 \tau}\right)\left[\tau\left(S+|d f|^{2}\right)+f-n\right] d m .
\end{aligned}
$$

Since we think of $\tau$ as backward time, we set $\sigma \equiv-1$. As before we choose the variation of $f$ such that the measure is kept fixed:

$$
h:=\frac{\operatorname{tr} v}{2}+\frac{n}{2 \tau} \Rightarrow \frac{\operatorname{tr} v}{2}-h+\frac{n}{2 \tau}=0 .
$$

Fix $f$ and choose $h$ as above. In the same way fix $\tau$ and choose $\sigma$ as above. Considering $W$ as a functional of $g$ and $u$ alone, we finally get:

$$
\begin{aligned}
\delta W[g, u, f, \tau](v, w) & \\
= & \int_{M} v_{i j} \cdot\left\{-\tau S_{i j}-\tau \nabla_{i} \nabla_{j} f\right\}+w \cdot 2 \alpha_{n} \tau\{\Delta u-\langle d u, d f\rangle\} d m \\
& +\int_{M}[\tau \cdot \frac{n}{2 \tau} \underbrace{\left(2|d f|^{2}-2 \Delta f\right)}_{=0}+h-S-\underbrace{|d f|^{2}}_{=\Delta f}] d m .
\end{aligned}
$$

Since the following identity is valid on closed $M$

$$
0=(4 \pi \tau)^{-n / 2} \int_{M} \Delta e^{-f} d V=\int_{M}\left(|d f|^{2}-\Delta f\right)(4 \pi \tau)^{-n / 2} e^{-f} d V
$$


we can cancel one term in (6.1) and replace $|d f|^{2}$ by $\Delta f$ in the other. If we deform $W$ along the variation given by the evolution equations

$$
\begin{aligned}
v & :=\partial_{t} g:=-2 S y-2 \nabla^{2} f, \\
w & :=\partial_{t} u:=\Delta u-\langle d u, d f\rangle \\
h & :=\partial_{t} f:=\frac{\operatorname{tr} v}{2}+\frac{n}{2 \tau}=-\Delta f-S+\frac{n}{2 \tau}, \\
\sigma & :=\partial_{t} \tau:=-1,
\end{aligned}
$$

we calculate that

$$
\begin{aligned}
& \partial_{t} W(g, u, f, \tau)(t) \\
& \quad=\int_{M}\left[2 \tau\left|S y+\nabla^{2} f\right|^{2}+2 \alpha_{n} \tau|\Delta u-\langle d u, d f\rangle|^{2}-2 \Delta f-2 S+\frac{n}{2 \tau}\right] d m .
\end{aligned}
$$

Since

$$
2 \tau\left|S y+\nabla^{2} f-\frac{1}{2 \tau} g\right|^{2}=2 \tau\left|S y+\nabla^{2} f\right|^{2}+2 \tau\left(\frac{n}{4 \tau^{2}}-\frac{1}{\tau} S-\frac{1}{\tau} \Delta f\right),
$$

everything comes together to the following result:

Theorem 6.1. Let $M$ be a closed Riemannian manifold and $(g, u, f, \tau)(t)$ a solution on $[0, T) \times M$ of the evolution equations

$$
\begin{aligned}
& \partial_{t} g=-2 S y, \\
& \partial_{t} u=\Delta u, \\
& \partial_{t} f=-\Delta f+|\nabla f|^{2}-S+\frac{n}{2 \tau}, \\
& \partial_{t} \tau=-1 .
\end{aligned}
$$

Then the following monotonicity formula holds:

$$
\partial_{t} W(t)=\int_{M}\left[2 \tau\left|S y+\nabla^{2} f-\frac{1}{2 \tau} g\right|^{2}+2 \alpha_{n} \tau|\Delta u-d u(\nabla f)|^{2}\right] d m \geq 0 .
$$

In particular, the entropy $W$ is nondecreasing. Equality holds if and only if $(g, u, f, \tau)(t)$ satisfies

$$
S y+\nabla^{2} f-\frac{1}{2 \tau} g=0 \quad \text { and } \quad \Delta u-d u(\nabla f)=0 .
$$

Such a solution is called a homothetic shrinking gradient soliton. 
Proof. We apply the diffeomorphisms generated by $\nabla f(t)$ to the system (6.2) in the same way as we did for (3.3). The result follows considering that $W$ is invariant under diffeomorphisms. More details are given in [15, §4].

Remark 6.2. Note that the theorem is still true for a complete noncompact manifold $\Sigma$ as long as the integrations by parts can be justified. This is possible for example by imposing decay conditions on $(g, u, f)$.

We derive a useful quantity from $W$.

Definition 6.3. Let $(g, u, \tau) \in \mathcal{M}(M) \times C^{\infty}(M) \times \mathbb{R}^{+}$be given. Then we define:

$$
\mu:=\mu(g, u, \tau):=\inf _{f \in C^{\infty}(M)}\left\{W(g, u, f, \tau): \int_{M}(4 \pi \tau)^{-n / 2} e^{-f} d V=1\right\} .
$$

Proposition 6.4. Let $M$ be closed and connected. Then $\mu$ is attained by a smooth function $\bar{f} \in C^{\infty}(M)$ satisfying the normalization constraint.

Proof. We adapt the method from [23] to our situation and refer to the proof of [15, Proposition 5.8] for the technical details.

Remark 6.5. $W$ is still bounded below on complete $\Sigma$, but a weakly convergent minimizing sequence in $W^{1,2}$ need not converge strongly in any $L^{p}$ space, i.e., the support of the functions in the sequence could become infinite or move out to infinity. It should be possible to find a contradiction to this scenario on asymptotically flat manifolds. A noncollapsing result analogous to Theorem 7.2 would then follow immediately.

Lemma 6.6. Suppose $(g, u)(t)$ is a solution to (3.1) on $[0, T) \times M$ where $M$ is closed. Fix $a \bar{\tau} \in[0, T)$ and define $\tau(t):=\bar{\tau}-t$. Then $\mu(g, u, \tau)(t)$ is nondecreasing in $t$. If $\frac{d}{d t} \mu(t)=0$, the solution is a gradient shrinking soliton.

Proof. Fix $t_{0}$ and let $\bar{f}$ be a minimizer for $\mu$ at time $t_{0}$. Solving $\partial_{t} f=$ $-\Delta f-S+\frac{n}{2 \tau}$ backwards in time with initial data $\bar{f}$ at $t_{0}$, we conclude from (6.3) for all $t<t_{0}$ that

$$
\mu(t) \leq W(g, u, f, \tau)(t) \leq W\left(g\left(t_{0}\right), u\left(t_{0}\right), \bar{f}, \tau(0)\right)=\mu\left(t_{0}\right) .
$$

Therefore, $\mu(t)$ is nondecreasing in time. The equality case follows from the equality case for $W$. 
We use the monotonicity formula to prove bounds on the injectivity radius of a solution. Another application, which is motivated by Perelman [7], is the proof of nonexistence of periodic solutions, so-called breathers. We just state the result and skip the details.

Theorem 6.7. Let $(g, u)(t)$ be a steady/expanding/shrinking breather on a closed manifold $M$. Then it necessarily is a steady/expanding/shrinking gradient soliton. In the steady case, $(M, g)$ is Ricci-flat and $u$ is constant. In the expanding case, $(M, g)$ is Einstein and $u$ is constant.

Proof. The complete proof is given in $[15, \S 5]$.

\section{Finite-time singularities}

In general, solutions will only exist for a certain finite time due to curvature blowup. To be able to study singularity formation, it is absolutely crucial to keep control on the injectivity radius when rescaling the solution.

Definition 7.1. A solution $(g, u)(t)$ is locally collapsing at $T$, if there are sequences of times $\left(t_{k}\right), t_{k} \rightarrow T$ and balls $\left(B_{k}\right):=\left(B_{r_{k}}\left(p_{k}\right)\right)$ at time $t_{k}$ such that $r_{k}^{2} / t_{k}$ is bounded, the curvature satisfies $|R m|\left(t_{k}\right) \leq r_{k}^{-2}$ on $B_{k}$, and the volume decreases like $r_{k}^{-n} \operatorname{vol}\left(B_{k}\right) \rightarrow 0$.

Inspired by Perelman's construction [7, §4], we prove:

Theorem 7.2. Suppose $M$ is closed and $T<\infty$. Then a solution $(g, u)(t)$ of (3.1) on $[0, T)$ is noncollapsed on $[0, T] \times M$.

Proof. Assume to the contrary the existence of sequences $\left(t_{k}\right)$ and $\left(B_{k}\right)$ as above. Setting $\phi:=e^{-f / 2}$, we realize that $\mu(g, u, \tau)(t)$ is the infimum of

$$
\tilde{W}(g, u, w, \tau):=\int_{M}\left[\tau\left(4|d \phi|^{2}+S \phi^{2}\right)-\phi^{2} \ln \phi^{2}-n \phi^{2}\right](4 \pi \tau)^{-n / 2} d V
$$

given the constraint

$$
\int_{M} \phi^{2}(4 \pi \tau)^{-n / 2} d V=1
$$

Set $\tau(t):=\left(t_{k}+r_{k}^{2}\right)-t$ and define a test function $\phi_{k}(x):=e^{C_{k}} \xi\left(r_{k}^{-1}\right.$ $\left.d\left(x, p_{k}\right)\right)$ at time $t_{k}$ where $\xi \in C^{\infty}\left(\mathbb{R}^{+}\right)$satisfies $\xi \equiv 1$ on $\left[0, \frac{1}{2}\right], \xi \searrow$ on $\left[\frac{1}{2}, 1\right]$ and $\xi \equiv 0$ on $[0, \infty)$. Choose $C_{k}$ such that the normalization condition (7.2) 
for $\phi_{k}$ is satisfied. Noting that $\phi_{k}$ vanishes outside $B_{k}$ and that $\tau\left(t_{k}\right)=r_{k}^{2}$, we compute

$$
(4 \pi)^{n / 2}=r_{k}^{-n} \int_{M} \phi_{k}^{2} d V=e^{2 C_{k}} r_{k}^{-n} \int_{B_{k}} \xi^{2} d V \leq e^{2 C_{k}} \underbrace{r_{k}^{-n} \operatorname{vol}\left(B_{k}\right)}_{\longrightarrow 0}
$$

which forces $C_{k} \rightarrow+\infty$ for $k \rightarrow \infty$. We insert $\phi_{k}$ and $r_{k}^{2}$ into (7.1) and get

$$
\begin{aligned}
\tilde{W}\left(g, u, \phi_{k}, r_{k}^{2}\right)= & \left.(4 \pi)^{-n / 2} r_{k}^{-n} e^{2 C_{k}} \int_{B_{k}} 4 r_{k}^{2}\left|\xi^{\prime}\right|^{2} \cdot\left(r_{k}^{-2}|\nabla d|^{2}\right)-2 \xi^{2} \ln \xi\right) d V \\
& +r_{k}^{2} \int_{B_{k}} S \phi_{k}^{2}(4 \pi)^{-n / 2} r_{k}^{-n} d V-n-2 C_{k} \\
\leq & (4 \pi)^{-n / 2} r_{k}^{-n} e^{2 C_{k}} \int_{B_{k}}\left(4\left|\xi^{\prime}\right|^{2}-2 \xi^{2} \ln \xi\right) d V \\
& +r_{k}^{2} \max _{B_{k}} S-n-2 C_{k} .
\end{aligned}
$$

Set $V(r):=\operatorname{vol}\left(B_{r_{k}}\left(p_{k}\right)\right)$. Using the curvature bound $R c \geq-(n-1) C^{2} r_{k}^{-2}$ on $B_{k}$, we can compare $V(r)$ with the volume $\tilde{V}(r)$ of the ball in the model space of negative constant sectional curvature. The Bishop Volume Comparison Theorem [20, Theorem 1.3] implies that

$$
V\left(r_{k}\right)-V\left(r_{k} / 2\right) \leq\left(C^{\prime}-1\right) V\left(r_{k} / 2\right)
$$

Since $\xi \equiv 1$ on $B_{r_{k} / 2}\left(p_{k}\right)$, this allows us to estimate

$$
\begin{aligned}
\int_{B_{k}}\left(4\left|\xi^{\prime}\right|^{2}-2 \xi^{2} \ln \xi\right) d V & \leq\left.\max _{r_{k} / 2 \leq d\left(p_{k}, x\right) \leq r_{k}}|4| \xi^{\prime}\right|^{2}-2 \xi^{2} \ln \xi \mid \cdot\left[V\left(r_{k}\right)-V\left(r_{k} / 2\right)\right] \\
& \leq C \cdot V\left(r_{k} / 2\right) \leq C \int_{B_{k}} \xi^{2} d V=C(4 \pi)^{n / 2} r_{k}^{n} e^{-2 C_{k}}
\end{aligned}
$$

Plugging this into (7.3), we conclude for a constant $\tilde{C}$ independent of $k$ :

$$
\begin{aligned}
\tilde{W}\left(g, u, \phi_{k}, r_{k}^{2}\right) & \leq C+r_{k}^{2} \max _{B_{k}}\left(R-2|d u|^{2}\right)-n-2 C_{k} \\
& \leq C+r_{k}^{2} \max _{B_{k}} R-2 C_{k} \\
& \leq \tilde{C}-2 C_{k} \stackrel{k \rightarrow \infty}{\longrightarrow}-\infty
\end{aligned}
$$

where we estimated $\max R \leq n^{2} \cdot r_{k}^{-2}$ by the initial bound on $R m$. Choosing $\tau(t)=\left(t_{k}+r_{k}^{2}\right)-t$, the monotonicity of $\mu$ from Lemma 6.6 shows for 
$k \rightarrow \infty:$

$\mu\left(g(0), u(0), t_{k}+r_{k}^{2}\right) \leq \mu\left(g\left(t_{k}\right), u\left(t_{k}\right), r_{k}^{2}\right) \leq \tilde{W}\left(g\left(t_{k}\right), u\left(t_{k}\right), \phi_{k}, r_{k}^{2}\right) \longrightarrow-\infty$.

But $\mu(0)>-\infty$ since $g(0)$ and $u(0)$ are fixed and smooth and $t_{k}+r_{k}^{2}$ is bounded.

The notion of noncollapse can be made more precise:

Definition 7.3. A metric $g$ is said to be $\kappa$-noncollapsed on the scale $\rho$, if every metric ball $B_{r}\left(x_{0}\right)$ of radius $r<\rho$ such that $\sup _{x \in B_{r}\left(x_{0}\right)}|R m| \leq r^{-2}$ has volume at least $\kappa r^{n}$.

Thus Theorem 7.2 implies on closed $M$ that $g(t)$ is $\kappa$-noncollapsed on the scale $\sqrt{T}$ for all $t \in[0, T)$ and some $\kappa$ depending only on $n, \sup _{M}|\tilde{R} m|_{0}$, $\operatorname{inj}(\tilde{g})$, and $T$. Note that control on the volume of small balls is sufficient for a lower bound on the injectivity radius of $M[24$, Theorem 4.7] and [15, Proposition 7.7].

To be able to speak of compactness for solutions to (3.1), we need to define convergence for sequences of such solutions:

Definition 7.4. Let $\left(g_{k}, u_{k}\right)(t)$ be a family of solutions to (3.1) on $\left[T_{A}, T_{O}\right) \times \Sigma_{k}$ where $\Sigma_{k}$ is complete. Let $x_{k}$ be a base point in $\Sigma_{k}$. Furthermore, let $\Sigma_{\infty}$ be a complete Riemannian manifold, $\left(g_{\infty}, u_{\infty}\right)(t)$ a solution to (3.1), and $x_{\infty} \in \Sigma_{\infty}$ be a base point. Then the sequence $\left(\Sigma_{k},\left(g_{k}, u_{k}\right)(t), x_{k}\right)$ converges to $\left(\Sigma_{\infty},\left(g_{\infty}, u_{\infty}\right)(t), x_{\infty}\right)$, if there exists a sequence of open sets $U_{k} \subset \Sigma_{\infty}$ containing $x_{\infty}$, and a sequence of diffeomorphisms $F_{k}: U_{k} \rightarrow V_{k}$ where $V_{k} \subset \Sigma_{k}$ is open, satisfying $F_{k}\left(x_{\infty}\right)=x_{k}$ such that any compact set in $\Sigma_{\infty}$ eventually lies in all $U_{k}$ and the pullbacks $\tilde{g}_{k}(t):=F_{k}^{*} g_{k}(t)$ and $\tilde{u}_{k}(t):=$ $F_{k}^{*} u_{k}(t)$ converge to $\left(g_{\infty}, u_{\infty}\right)(t)$ on every compact subset of $\left(T_{A}, T_{O}\right) \times \Sigma_{\infty}$ uniformly together with all their derivatives.

Using the interior estimates in Theorem 5.12, we can prove compactness of a set of solutions.

Theorem 7.5. Let $T_{A}, T_{O}$ be given such that $-\infty \leq T_{A}<T_{O} \leq \infty$. Fix $t_{0} \in\left(T_{A}, T_{O}\right)$. Let $\left(\Sigma_{k}, g_{k}(t), u_{k}(t), x_{k}\right)$ be a pointed sequence of complete 
solutions to (3.1) for $t \in\left[T_{A}, T_{O}\right)$ with

$$
\begin{gathered}
\sup _{\Sigma_{k}}\left|R m_{k}\right|_{g_{k}(t)}(t) \leq C_{0} \quad \forall t \in\left(T_{A}, T_{O}\right) \\
\sup _{\Sigma_{k}}\left|u_{k}\right|\left(T_{A}\right) \leq C_{0}^{\prime}
\end{gathered}
$$

where $C_{0}$ and $C_{0}^{\prime}$ are independent of $k$. Assume in addition that $\left(g_{k}, u_{k}\right)\left(t_{0}\right)$ is $\kappa$-noncollapsed for some $\kappa>0$ independent of $k$. Then there exists a subsequence

$$
\left(\Sigma_{k}, g_{k}(t), u_{k}(t), x_{k}\right) \stackrel{C^{\infty}}{\longrightarrow}\left(\Sigma_{\infty}, g_{\infty}(t), u_{\infty}(t), x_{\infty}\right)
$$

converging to a complete, $\kappa$-noncollapsed solution of (3.1). All derivatives of the curvature $R m_{\infty}$ and of $u_{\infty}$ are bounded above and there is a lower bound on the injectivity radius of $g_{\infty}$.

We need an auxiliary lemma similar to [4, Lemma 2.4] to get estimates for the solutions $\left(g_{k}, u_{k}\right)(t)$ with respect to the limit metric $g_{\infty}(t)$ using bounds at $t=t_{0}$ and bounds for $g_{k}(t), u_{k}(t)$ with respect to the metrics $g_{k}(t)$.

Lemma 7.6. Let $(\Sigma, g)$ be a Riemannian manifold and $K \subset \Sigma$ be a compact subset. Assume $\left(g_{k}, u_{k}\right)(t)$ is a collection of solutions to (3.1), defined on neighborhoods of $[\alpha, \omega] \times K$ such that $\alpha<0<\omega$. Let $\nabla,|\cdot|$ denote covariant differentiation and length with respect to $g$ and ${ }^{k} \nabla,|\cdot|_{k}$ with respect to $g_{k}$. Furthermore, suppose that at time $t=0$ on $K$ we have the bounds

(a) $c g(X, X) \leq g_{k}(X, X) \leq C g(X, X)$ for all $X \in \mathcal{X}(\Sigma)$,

(b) $\left|\nabla^{p+1} g_{k}\right| \leq \hat{C}_{p+1}, \quad\left|\nabla^{p} u_{k}\right| \leq \hat{C}_{p}^{\prime}$ for all $p \geq 0$,

and in addition

(c) $\sup _{[\alpha, \omega] \times K}\left(\left|{ }^{k} \nabla^{p} R m_{k}\right|_{k}+\left|{ }^{k} \nabla^{p} u_{k}\right|_{k}\right) \leq C_{p}+C_{p}^{\prime}$ for all $p \geq 0$

with constants $c, C, \hat{C}_{p}, \hat{C}_{p}^{\prime}, C_{p}, C_{p}^{\prime}$ independent of $k$. Then the following holds:

(i) $\tilde{c} g(X, X) \leq g_{k}(X, X) \leq \tilde{C} g(X, X)$ on $[\alpha, \omega] \times K$,

(ii) $\sup _{[\alpha, \omega] \times K}\left(\left|\nabla^{p+1} g_{k}\right|+\left|\nabla^{p} u_{k}\right|\right) \leq \tilde{C}_{p+1}+\tilde{C}_{p}^{\prime}$ for all $p \geq 0$

for constants $\tilde{c}, \tilde{C}, \tilde{C}_{p}, \tilde{C}_{p}^{\prime}$ independent of $k, \alpha, \omega$ and $K$. 
Proof. From $(a)$ and $(c)$ we obtain the equivalence of the metrics $g_{k}(t)$ to $g_{k}(0)$ and therefore also to $g$ analogously to [4, Lemma 2.4] for constants $\tilde{c}$ and $\tilde{C}$ depending only on $n, C_{0}, \hat{C}_{1}^{\prime}, T$ and $C$. Denote by $Z$ from now on all constants depending only on $\tilde{c}, \tilde{C}$.

The evolution (3.5) of $\Gamma_{k}$ together with $(c)$ yields the estimate:

$$
\left|\partial_{t}\left(\Gamma_{k}-\Gamma\right)\right|_{k} \leq\left.\left. c(n)\right|^{k} \nabla R c_{k}\right|_{k}+\left.\left.c(n)\left|d u_{k}\right|_{k}\right|^{k} \nabla^{2} u_{k}\right|_{k} \leq C_{1}+\hat{C}_{1} \hat{C}_{2}=: A_{1}
$$

for a constant $A_{1}=A_{1}\left(n, C_{1}, \hat{C}_{1}, \hat{C}_{2}\right)$. Since $\nabla g_{k} \simeq \Gamma_{k}-\Gamma \simeq{ }^{k} \nabla-\nabla$, we deduce

$$
\left|\partial_{t} \nabla g_{k}\right| \leq c(n)\left|\partial_{t}\left(\Gamma_{k}-\Gamma\right)\right| \leq c(n) Z\left|\partial_{t}\left(\Gamma_{k}-\Gamma\right)\right|_{k} \leq Z \cdot A_{1}
$$

Using the bounds $(b)$ on $\nabla g_{k}$ at $t=0$, an integration gives

$$
\begin{aligned}
\left|\nabla g_{k}\right|(t) & =\left|\nabla g_{k}(0)+\int_{0}^{t} \partial_{\tau} \nabla g_{k}(\tau) d \tau\right| \leq\left|\nabla g_{k}\right|(0)+Z A_{1} T \\
& \leq \hat{C}_{1}+Z A_{1} T=: \tilde{C}_{1} .
\end{aligned}
$$

Since $u_{k}$ is bounded on $[\alpha, \omega] \times K$ by $(c)$ and $|\cdot|=|\cdot|_{k}$ on functions, we easily obtain

$$
\left|u_{k}\right|(t)=\left|u_{k}\right|_{k}(t) \leq C_{0}=: \tilde{C}_{0}^{\prime}
$$

Similarly, $(c)$ and $(i)$ yield for the differential

$$
\left|\nabla u_{k}\right|(t)=\left|d u_{k}\right|(t)=\left|{ }^{k} \nabla u_{k}\right|(t) \leq\left.\left. Z \cdot\right|^{k} \nabla u_{k}\right|_{k}(t) \leq Z \cdot C_{1}=: \tilde{C}_{1}^{\prime} .
$$

Since we already have a bound for $\nabla g_{k}$ on $[\alpha, \omega] \times K$, we can estimate $\nabla^{2} u$ at time $t$ as follows:

$$
\partial_{t} \nabla^{2} u_{k}=\nabla^{2} f_{k}=\left(\nabla-{ }^{k} \nabla\right) d f_{k}+{ }^{k} \nabla d f_{k}=\nabla g_{k} *{ }^{k} \nabla f_{k}+{ }^{k} \nabla^{2} f_{k}
$$

where $f_{k}(t):=\Delta^{k} u_{k}(t) \in C^{\infty}\left(\left[T_{A}, T_{O}\right) \times \Sigma\right)$ is just an abbreviation. Therefore, we get

$$
\left|\partial_{t} \nabla^{2} u_{k}\right| \leq\left.\left.\left|\nabla g_{k}\right| Z\right|^{k} \nabla f_{k}\right|_{k}+\left.\left.Z\right|^{k} \nabla^{2} f_{k}\right|_{k} \leq \tilde{C}_{1} Z C(n) C_{3}^{\prime}+Z C(n) C_{4}^{\prime}=: B_{2}
$$

from $(c)$. Using $(b)$, an integration gives for $\tilde{C}_{2}^{\prime}=\tilde{C}_{2}^{\prime}\left(n, \tilde{c}, \tilde{C}, \tilde{C}_{1}, C_{3}^{\prime}, C_{4}^{\prime}, T\right)$ :

$$
\left|\nabla^{2} u_{k}\right|(t) \leq\left|\nabla^{2} u_{k}\right|(0)+\int_{0}^{t}\left|\partial_{\tau} \nabla^{2} u_{k}(\tau)\right| d \tau \leq \hat{C}_{2}^{\prime}+B_{2} T=: \tilde{C}_{2}^{\prime} .
$$

Higher derivatives of $\left(g_{k}, u_{k}\right)$ with respect to $g$ can be estimated in pairs $\left(\nabla^{p} g_{k}, \nabla^{p+1} u_{k}\right)[15,8.10]$. The technique is similar for all $p \geq 2$, so we only 
state the case $p=2$ as reference. Since $\nabla$ commutes with $\partial_{t}$, we get an expression for $\partial_{t} \nabla^{p} g_{k}$ from the flow equations (3.1):

$$
\begin{aligned}
\partial_{t} \nabla^{2} g_{k} & =\nabla^{2}\left(-2 R c_{k}+2 \alpha_{n} d u_{k} \otimes d u_{k}\right) \\
& =\nabla^{2} R c_{k}+\nabla^{3} u_{k} * d u_{k}+\nabla^{2} u_{k} * \nabla^{2} u_{k} .
\end{aligned}
$$

Using ${ }^{k} \nabla-\nabla \simeq \nabla g_{k}$, this can be rewritten in the following way:

$$
\begin{aligned}
\nabla^{2} R c_{k}= & \nabla g_{k} * \nabla g_{k} * R c_{k}+\nabla g_{k} *{ }^{k} \nabla R c_{k}+{ }^{k} \nabla \nabla g_{k} * R c_{k} \\
& +\nabla g_{k} *{ }^{k} \nabla R c_{k}+{ }^{k} \nabla^{2} R c_{k} .
\end{aligned}
$$

From the identity ${ }^{k} \nabla \nabla g_{k}=\nabla^{2} g_{k}+\nabla g_{k} * \nabla g_{k}$, we get altogether:

$$
\begin{aligned}
\partial_{t} \nabla^{2} g_{k}= & \nabla^{2} g_{k} * R c_{k}+\nabla^{3} u_{k} * \nabla u_{k}+\nabla g_{k} * \nabla g_{k} * R c_{k}+\nabla g_{k} *{ }^{k} \nabla R c_{k} \\
& +{ }^{k} \nabla^{2} R c_{k}+\nabla^{2} u_{k} * \nabla^{2} u_{k} .
\end{aligned}
$$

Therefore, it is necessary to control $\nabla^{3} u_{k}$ to get an estimate for $\nabla^{2} g_{k}$. Keeping this in mind, we estimate using $(c)$ :

$$
\begin{aligned}
\left|\partial_{t} \nabla^{2} g_{k}\right| & \leq Z C_{0}\left|\nabla^{2} g_{k}\right|+Z \tilde{C}_{1}^{\prime}\left|\nabla^{3} u_{k}\right|+Z\left\{\tilde{C}_{1}^{2} C_{0}+\tilde{C}_{1} C_{1}+C_{2}+\left(\tilde{C}_{2}^{\prime}\right)^{2}\right\} \\
& \leq A_{1}\left(\left|\nabla^{2} g_{k}\right|+\left|\nabla^{3} u_{k}\right|\right)+A_{2},
\end{aligned}
$$

defining $A_{1}:=Z \max \left\{C_{0}, \tilde{C}_{1}^{\prime}\right\} \quad$ and $\quad A_{2}=A_{2}\left(n, \tilde{c}, \tilde{C}, C_{0}, C_{1}, C_{2}, \tilde{C}_{1}, \tilde{C}_{2}^{\prime}\right)$. Doing the same calculation for $\nabla^{3} u_{k}$, we get

$$
\partial_{t} \nabla^{3} u_{k}=\left(\nabla g_{k} * \nabla g_{k}+\nabla^{2} g_{k}+\nabla g_{k} * \nabla g_{k}\right) *{ }^{k} \nabla f_{k}+\nabla g_{k} *{ }^{k} \nabla^{2} f_{k}+{ }^{k} \nabla^{3} f_{k} .
$$

This leads to the estimate (again using $(c)$ ):

$$
\begin{aligned}
\left|\partial_{t} \nabla^{3} u_{k}\right| & \leq Z C(n) C_{3}^{\prime}\left|\nabla^{2} g_{k}\right|+C(n) Z\left\{\tilde{C}_{1}^{2} C_{3}^{\prime}+\tilde{C}_{1} C_{4}^{\prime}+C_{5}^{\prime}\right\} \\
& \leq A_{3}\left|\nabla^{2} g_{k}\right|+A_{4}
\end{aligned}
$$

where $A_{3}=A_{3}\left(n, \tilde{c}, \tilde{C}, C_{3}^{\prime}\right)$ and $A_{4}=A_{4}\left(n, \tilde{c}, \tilde{C}, \tilde{C}_{1}, C_{3}^{\prime}, C_{4}^{\prime}, C_{5}^{\prime}\right)$. Putting (7.8) and (7.9) together and realizing that $|\cdot|$ is independent of time, we arrive at

$$
\begin{aligned}
\left|\partial_{t}\left(\left|\nabla^{2} g_{k}\right|+\left|\nabla^{3} u_{k}\right|\right)\right| & =|| \partial_{t} \nabla^{2} g_{k}|+| \partial_{t} \nabla^{3} u_{k}|| \\
& \leq A_{1}\left(\left|\nabla^{2} g_{k}\right|+\left|\nabla^{3} u_{k}\right|\right)+A_{2}+A_{3}\left|\nabla^{2} g_{k}\right|+A_{4} \\
& \leq B_{1}\left(\left|\nabla^{2} g_{k}\right|+\left|\nabla^{3} u_{k}\right|\right)+B_{2}
\end{aligned}
$$


We can use $(b)$ to integrate the equation in time as before and obtain

$$
\left|\nabla^{2} g_{k}\right|(t)+\left|\nabla^{3} u_{k}\right|(t) \leq \tilde{C}_{2}=\tilde{C}_{3}^{\prime}
$$

Here both constants depend only on $n, \tilde{c}, \tilde{C}, \hat{C}_{2}, \hat{C}_{3}^{\prime}, \tilde{C}_{1}, C_{0}, C_{1}, C_{2}, C_{3}^{\prime}, C_{4}^{\prime}, C_{5}^{\prime}$ and $T$. Together with (7.5), (7.6), (7.7) and the analogs of (7.10) for $p>2$, (ii) follows.

Proof of Theorem 7.5. Assume without loss of generality that $T_{A}, T_{O}<\infty$ and $t_{0}=0$. Let $\left(g_{k}, u_{k}\right)(t)$ be a sequence of solutions on $\left[T_{A}, T_{O}\right) \times \Sigma_{k}$ such that $\left|R m_{k}\right|_{k}^{2}(t) \leq C_{0}$. Then we can bound the injectivity radius $\operatorname{inj}\left(g_{k}(0)\right)>$ $\delta$ at time $t=0$ uniformly in $k$ from the $\kappa$-noncollapsing assumption $(\kappa$ is also uniform in $k$ ) using [24, Theorem 4.7] and the uniform curvature bound.

The uniform bound $\left|u_{k}\left(T_{A}\right)\right| \leq C_{0}^{\prime}$ from (7.4) implies not only a uniform bound $\left|u_{k}\right| \leq C_{0}^{\prime}$ on $\left[T_{A}, T_{O}\right) \times \Sigma$ from (5.11), but also a uniform bound $\left|d u_{k}\right|_{k}^{2} \leq C_{1}^{\prime}=C_{1}^{\prime}\left(C_{0}, T\right)$, using (5.7). Here $C_{1}^{\prime}$ only depends on $C_{0}^{\prime}$ and $T$. Therefore, we can apply Theorem 5.12 to get the uniform bounds

$$
\sup _{\left(T_{A}, T_{O}\right) \times \Sigma_{k}}\left(\left|{ }^{k} \nabla^{i} R m_{k}\right|_{k}+\left.\left.\right|^{k} \nabla^{i+2} u_{k}\right|_{k}\right) \leq C_{i}=C_{i}\left(n, T, C_{0}, C_{0}^{\prime}\right)
$$

for all $i \geq 0$, where the $C_{i}$ are constants depending only on the curvature bound $C_{0}$, the initial bound on $u_{k}$ given by $C_{0}^{\prime}, n$ and $T$, but not on $k$.

Using these bounds at $t=0$ and the lower injectivity radius bound, we can apply [2, Theorem 16.1] to get a convergent subsequence of $\left(\Sigma_{k}, g_{k}(0)\right.$, $x_{k}$ ) at time $t=0$ to a limit $\left(\Sigma_{\infty}, G, x_{\infty}\right)$. Note that the convergence is with respect to the limit metric $G$. The pullbacks $\tilde{g}_{k}:=F_{k}^{*} g_{k}(t)$ and $\tilde{u}_{k}:=$ $F_{k}^{*} u_{k}(t)$ are defined for all times $t \in\left(T_{A}, T_{O}\right)$ though. To prove convergence of $g_{k}, u_{k}$ for all $t$, we need uniform estimates for the derivatives of $\tilde{g}_{k}, \tilde{u}_{k}$ on $\left(T_{A}, T_{O}\right) \times \Sigma_{\infty}$. These estimates follow from Lemma 7.6 on all compact subsets $[\alpha, \omega] \times K \subset\left(T_{A}, T_{O}\right) \times \Sigma$. Therefore, we can find a subsequence converging uniformly on every compact subset of $\left(T_{A}, T_{O}\right) \times \Sigma_{\infty}$. The limit $g_{\infty}(t):=\lim _{k \rightarrow \infty} \tilde{g}_{k}(t)$ will agree at time $t=0$ with $G$ since it already converged there by construction. Defining $u_{\infty}(t):=\lim _{k \rightarrow \infty} \tilde{u}_{k}(t)$, we see that $\left(g_{\infty}, u_{\infty}\right)(t)$ is also a solution of (3.1) since the convergence is smooth and taking the limit commutes with all derivatives. Furthermore, it satisfies the same bounds on derivatives and the injectivity radius. If $T_{A}=\infty$ or $T_{O}=\infty$, we apply the theorem for a sequence of times $T_{A_{j}} \rightarrow-\infty$ or $T_{O_{j}} \rightarrow \infty$, respectively, on finite time intervals. A diagonalization argument yields a subsequence converging on the union of these intervals $[4, \S 2]$. 
Due to the noncollapsing result in Theorem 7.2, we can rescale the solution at a singular time. This can be seen as a microscopic view on the solution when approaching the singularity. Then a comparison of the solution near the singular time and close enough to the singular point with the rescaling limit is possible. It is crucial to know what these regions look like to set up the delicate surgery procedures as described in [5] or [7]. We first give some definitions.

Definition 7.7. A solution $(g, u)(t)$ to $(3.1)$ on a complete Riemannian manifold is called ancient, if it exists for all $t \in(-\infty, T]$ up to some time $T \geq 0$.

Definition 7.8. Let $(g, u)(t)$ be a maximal solution to $(3.1)$ on $[0, T) \times \Sigma$ for some $T \in(0, \infty]$. A sequence $\left(t_{k}, x_{k}\right) \subset[0, T) \times \Sigma$ is called an essential blowup sequence, if $t_{i} \rightarrow T$, and there is a constant $C \geq 1$ such that $\sup _{\left[0, t_{k}\right] \times \Sigma}|R m|(t, x) \leq C|R m|\left(t_{k}, x_{k}\right)$.

Theorem 7.9. Let $(g, u)(t)$ be a solution to (3.1) on $[0, T) \times M$ for $M$ closed and $T$ finite. Assume $\left(t_{k}, x_{k}\right)$ is an essential blowup sequence and set $B_{k}:=|R m|\left(t_{k}, x_{k}\right)$. Define the rescalings

$$
g_{k}(s):=B_{k} \cdot g\left(s / B_{k}+t_{k}\right), \quad u_{k}(s):=u\left(s / B_{k}+t_{k}\right) .
$$

Then a subsequence of $\left(M, g_{k}(s), u_{k}(s), x_{k}\right)$ converges smoothly on compact subsets of $[0, T) \times M$ to a complete ancient solution $\left(M_{\infty}, g_{\infty}(s), u_{\infty}(s), x_{\infty}\right)$ which is noncollapsed on all scales for some $\kappa>0$. Moreover, $u_{\infty}(s) \equiv$ const and $g_{\infty}(s)$ is a solution to the Ricci Flow.

Proof. We want to apply Theorem 7.5 to the sequence of rescalings $\left(M, g_{k}(s)\right.$, $\left.u_{k}(s), x_{k}\right)$. By choice of the scale factor, the rescaled solution exists for $s \in\left[-B_{k} \cdot t_{k}, 0\right]$, and we can compute

$$
\sup _{x \in M}\left|R m_{k}\right|_{g_{k}}(s, x)=B_{k}^{-1} \sup _{x \in M}|R m|\left(s / B_{k}+t_{k}, x\right) \leq B_{k}^{-1} \cdot C B_{k}=C
$$

for all $s \in\left[-B_{k} \cdot t_{k}, 0\right]$ from the scaling behavior of $|R m|$. Since $u$ is controlled using (5.11), we get a uniform bound $\sup \left|u_{k}\right|(s) \leq C^{\prime}$ on $\left[-B_{k}\right.$. $\left.t_{k}, 0\right] \times M$ independent of $k$ since $u \in C^{\infty}(M)$ is scaling invariant. Finally, because $(g, u)(t)$ is defined on a finite time interval and on closed $M$, we know from Theorem 7.2 that it is $\kappa$-noncollapsed on the scale $\sqrt{T}$ for some $\kappa>0$ depending only on the initial data. A short calculation shows that the rescaled solutions $\left(g_{k}, u_{k}\right)(s)$ are also $\kappa$-noncollapsed for the same $\kappa$, 
but on larger and larger scales $\rho_{k}:=\sqrt{B_{k} T}$. (Remember that $B_{k} \rightarrow \infty$.) Therefore, we can apply Theorem 7.5 on all time intervals $[-A, 0], A>0$, to conclude the existence of a subsequence converging to a complete solution $\left(M_{\infty}, g_{\infty}(s), u_{\infty}(s), x_{\infty}\right)$. The limit is ancient since $-B_{k} \cdot t_{k} \rightarrow-\infty$ for $k \rightarrow \infty$ and $\kappa$-noncollapsed on all scales since $\rho_{k} \rightarrow \infty$.

It remains to show that the limit is in fact a solution to Ricci Flow. To this end, we will show that $\left|d u_{\infty}\right|_{g_{\infty}}^{2} \equiv 0$. Recall the a priori estimate (5.8) for $|d u|^{2}$ which is valid for all $t \in(0, T)$ :

$$
\sup _{x \in M}|d u|^{2}(t, x) \leq \frac{1}{2 \alpha_{n}} t^{-1}
$$

Since we have $g_{k}:=B_{k} \cdot g$, we get for all $s \in(-\infty, 0]$ and $k$ big enough that

$$
\begin{aligned}
\left|d u_{k}\right|_{g_{k}}^{2}(s) & =\left(g_{k}\right)^{i j} \partial_{i} u_{k} \partial_{j} u_{k}=B_{k}^{-1} \cdot|d u|^{2}\left(s / B_{k}+t_{k}\right) \leq \frac{1}{2 \alpha_{n}} B_{k}^{-1} \cdot \frac{B_{k}}{s+t_{k} B_{k}} \\
& =\frac{1}{2 \alpha_{n}} \cdot \frac{1}{s+t_{k} B_{k}}
\end{aligned}
$$

holds. Passing to the limit $k \rightarrow \infty$ for fixed $s$, we conclude that $\left|d u_{\infty}\right| g_{\infty}(s) \equiv$ 0 . Since $s$ is arbitrary, the system (3.1) reduces to the Ricci Flow equation for $g_{\infty}$ on $(-\infty, 0] \times M_{\infty}$.

The following immediate consequence is proven in [15, Corollary 8.7]:

Corollary 7.10. For every maximal solution $(g, u)(t)$ of the system $(3.1)$ on $[0, T) \times M$ for $T<\infty$ and closed $M$, there is a sequence of dilations such that the limit is a complete, ancient solution to the Ricci flow which is $\kappa$-noncollapsed on all scales for some $\kappa>0$.

Remark 7.11. Although we can always find an essential blowup sequence at a singular time $T$ of a given maximal solution $(g, u)(t)$, we point out that there may be other singularities forming at the same time but with a higher blowup rate. These are called slowly forming singularities. To fully understand the solution at time $T$, one also needs to understand these singularities. In $[2, \S 16]$ dilation limits at singularities are referred to as singularity models and are classified in two types I and $\mathrm{II}(\mathrm{a})$ for $T<\infty$. Following $[6, \S 11, \S 12]$, we do not make this distinction. Perelman instead uses "ancient $\kappa$-solutions" to study the Ricci flow singularities. 


\section{Asymptotically flat solutions}

We consider complete and asymptotically flat manifolds $(\Sigma, \tilde{g}, \tilde{u})$. This extends the usefulness of (3.1) to physical applications, in particular to the study of isolated systems. This class of solutions to the Einstein equations consists of metrics describing the gravitational field of a single body like a star or a black hole. The model for such a solution is an asymptotically flat manifold where the metric satisfies certain decay conditions near infinity. We prove for two definitions of asymptotic flatness that the decay behavior at time $t=0$ is maintained as long as the solution satisfies a supremum bound. However, the following estimate is valid for all solutions satisfying the stated bounds, for example for all solutions given by Theorem 4.1.

Lemma 8.1. Let $(g, u)(t)$ solve (3.1) on $[0, T] \times \Sigma$ with initial data $(\tilde{g}, \tilde{u})$ such that $|R m|+\left|\nabla^{2} u\right|+|d u|^{2} \leq \sqrt{K}$ on $[0, T] \times \Sigma$. Then for $t \in[0, T]$, any $x_{0} \in \Sigma$ and any $R>0$, the estimate

$$
\sup _{x \in B_{R / 4}^{t}\left(x_{0}\right)}|\Phi|^{2}(t, x) \leq 2 \sup _{x \in B_{R}^{0}\left(x_{0}\right)}|\Phi|_{0}^{2}(0, x) \cdot e^{C \tilde{C} t / R^{2}}
$$

holds, where $\tilde{C}:=\max \left\{R^{2} \cdot \sqrt{K}, 1\right\}$ is a scaling invariant constant and $C$ depends only on $n$.

Proof. The assumptions imply that $R^{2}\left(|\Phi|+|d u|^{2}\right) \leq R^{2} \cdot \sqrt{K}$ holds on $B\left(T, x_{0}, R\right)$. Set $\tilde{C}:=\max \left\{R^{2} \cdot \sqrt{K}, 1\right\}$. Using the evolution equation for $|\Phi|^{2}$ from Lemma 5.8, we get

$$
\left(\partial_{t}-\Delta\right)|\Phi|^{2} \leq-2|\nabla \Phi|^{2}+C(n) \tilde{C} R^{-2} \cdot|\Phi|^{2}
$$

Define the cut-off function $\tilde{\eta}(t, x):=R^{-4} \cdot\left(R^{2}-\frac{1}{2} d_{t}^{2}\left(x_{0}, x\right)\right)^{2}$ for a fixed radius $R>0$. It differs from the one defined in Lemma 5.5 only by the factor $R^{-4}$, and we therefore can use the results obtained in Lemma 5.5 for $\eta$ modified by this factor. Setting $f:=|\Phi|^{2} \cdot \tilde{\eta}$, we compute

$$
\begin{aligned}
\left(\partial_{t}-\Delta\right) f & \leq-2|\nabla \Phi|^{2} \tilde{\eta}+C(n) \tilde{C} R^{-2}\left\{f+|\Phi|^{2}\right\}+|\nabla \Phi|^{2} \tilde{\eta}+C(n) R^{-2} f \\
& \leq C(n) \tilde{C} R^{-2} f
\end{aligned}
$$


using the bound $\tilde{\eta}^{-1} \leq \frac{16}{9}$ on $B\left(T, x_{0}, R / 4\right)$ and $\tilde{C} \geq 1$. From $|\nabla d|=1$, we estimated

$$
\begin{aligned}
-2 \nabla|\Phi|^{2} \nabla \eta & \leq 4|\nabla \Phi||\Phi| \cdot \frac{\left(R^{2}-r\right)\left|\nabla d^{2}\right|}{R^{4}}=\left(|\nabla \Phi| \frac{R^{2}-d^{2}}{R^{2}} \cdot \frac{8|\nabla d||d||\Phi|}{R^{2}}\right) \\
& \leq|\nabla \Phi|^{2} \tilde{\eta}+C R^{-2}|\Phi|^{2} .
\end{aligned}
$$

The solution $v(t)=v(0) \cdot e^{C \tilde{C} t / R^{2}}$ to the associated ordinary differential equation with initial value $v(0):=\frac{9}{8} \sup _{x \in B_{R}^{0}\left(x_{0}\right)} f(0, x)$ is a barrier for $f(t)$ for all $t \geq 0$, and we therefore get

$$
\sup _{x \in B_{R / 4}^{t}\left(x_{0}\right)}\left[|\Phi|^{2}(t, x) \cdot \tilde{\eta}(t, x)\right] \leq \frac{9}{8} \sup _{x \in B_{R}^{0}\left(x_{0}\right)}\left[|\Phi|^{2}(0, x) \cdot \tilde{\eta}(0)\right] e^{C \tilde{C} t / R^{2}} .
$$

Using $\tilde{\eta}(0) \leq 1$ and $\tilde{\eta}^{-1} \leq \frac{16}{9}$ on $B\left(T, x_{0}, R / 4\right)$, the desired result follows.

Lemma 8.2. Let $(g, u)(t)$ be a solution to $(3.1)$ on $[0, T] \times \Sigma$ with initial data $(\tilde{g}, \tilde{u})$ such that $|R c|^{2} \leq K$ on $[0, T] \times \Sigma$. Then we get for $t \in[0, T]$, any $x_{0} \in \Sigma$ and any $R>0$ the estimate

$$
\sup _{x \in B_{R / 4}^{t}\left(x_{0}\right)}|d u|^{2}(t, x) \leq 2 \sup _{x \in B_{R}^{0}\left(x_{0}\right)}|d \tilde{u}|_{0}^{2}(0, x) \cdot e^{C \tilde{C} t / R^{2}},
$$

where $\tilde{C}:=\max \left\{R^{2} \cdot \sqrt{K}, 1\right\}$ is a scaling invariant constant and $C$ depends only on $n$.

Proof. Since $|d u|^{2}$ satisfies (3.6), a bound on the Ricci curvature is sufficient to apply (5.6). The remaining proof is analogous to the proof of Lemma 8.1 .

A strong definition of asymptotic flatness is given as follows:

Definition 8.3. Let $\Sigma$ be a complete $n$-dimensional connected Riemannian manifold. $(\Sigma, g, u)$ is called strongly asymptotically flat of mass $m$, if there is a compact subset $K \subset \Sigma$ such that $\Sigma_{K}:=\Sigma \backslash K$ is diffeomorphic to $\mathbb{R}^{n} \backslash$ $B_{1}(0)$ and $(g, u)$ satisfy in the exterior region $\Sigma_{K}$ :

$$
\begin{aligned}
\left|g-\left(1-\frac{2 m}{\tilde{r}}\right) \delta\right| & \leq C_{0} \cdot \tilde{r}^{-2}, \\
\left|\partial^{k} g\right| & \leq C_{k} \cdot \tilde{r}^{-k-1}, \quad k=1,2,3, \\
\left|u+\frac{m}{\tilde{r}}\right| & \leq D_{0} \cdot \tilde{r}^{-2}, \\
\left|\partial^{k} u\right| & \leq D_{k} \cdot \tilde{r}^{-k-1}, \quad k=1,2,3,
\end{aligned}
$$


for some constants $C_{k}, D_{k}, k=0, \ldots, 3$ where $\tilde{r}(x):=\sqrt{x_{1}^{2}+\cdots+x_{n}^{2}}$ is the radial coordinate of $\mathbb{R}^{n}$ and $|\cdot|$ and $\partial$ are with respect to $\delta$.

A very important physical property of an asymptotically flat manifold is its ADM mass. For manifolds as defined above, it is given by the coefficient $m$ in the expansion of $(g, u)$. The general definition can be found in [25] where it is also shown that the ADM mass is invariantly defined and independent of the asymptotic coordinate system. We prove that the flow (3.1) preserves the class of asymptotically flat manifolds as defined above. Observe that this theorem holds for all asymptotically flat solutions if uniqueness holds in Theorem 4.1 .

Theorem 8.4. Let $(g, u)(t)$ be the solution to $(3.1)$ on $[0, T] \times \Sigma$ for $T<\infty$ with initial data $(\tilde{g}, \tilde{u})$ given by Theorem 4.1. Assume that $(\Sigma, \tilde{g}, \tilde{u})$ is strongly asymptotically flat of mass $m:=m(\tilde{g})$ with $K=\bar{B}_{\rho}^{0}(O)$ for constants $C_{k}, D_{k}$. Then $(\Sigma, g(t), u(t))$ is strongly asymptotically flat for all $t \in[0, T]$. In particular, there is a constant $C(t)=C\left(t, k_{0}, c_{0}, s_{0}, C_{k}, D_{k}, n\right)$ depending only on time, the bounds from Theorem 4.1 and the asymptotic decay of $(\tilde{g}, \tilde{u})$ such that

$$
\begin{aligned}
\left|g(t)-\left(1-\frac{2 m}{\tilde{r}}\right) \delta\right|_{0}+\left|u(t)+\frac{m}{\tilde{r}}\right|_{0} & \leq C(t) \cdot \tilde{r}^{-2} \\
\left|\partial^{k} g\right|_{0}+\left|\partial^{k} u\right|_{0} & \leq C(t) \cdot \tilde{r}^{-k-1}
\end{aligned}
$$

holds on $[0, T] \times \Sigma \backslash B_{2 \rho}(O)$ for $k=1,2,3$ with $C(t) \rightarrow \infty$ for $t \rightarrow \infty$. In particular, the ADM mass is preserved by the flow and we have $m(g(t))=m$ for all $t \in[0, T]$.

Proof. Let $(\Sigma, \tilde{g}, \tilde{u})$ be asymptotically flat on $\Sigma_{K}:=\Sigma \backslash \bar{B}_{\rho}(O)$. Assume without loss of generality that $\rho \geq 1$. We know from the definition that there is a constant $\bar{C}$ such that

$$
\sup _{x \in \Sigma_{K}}\left[|\Phi|_{0}(0, x) \tilde{r}^{3}(x)\right] \leq C \sup _{x \in \Sigma_{K}}\left[\left(\left|\partial^{2} \tilde{g}\right|_{0}+|\partial \tilde{g}|_{0}^{2}+\left|\partial^{2} \tilde{u}\right|_{0}+|\partial \tilde{g}|_{0}|\partial \tilde{u}|_{0}\right) \tilde{r}^{3}\right] \leq \bar{C}
$$

Fix $x_{0} \in \Sigma \backslash B_{2 \rho}^{0}(O)$ and choose $R:=\frac{1}{4} \tilde{r}\left(x_{0}\right)$. Using the equivalence of the norms $|\cdot|_{0}$ and $|\cdot|$ and the bounds on $|\Phi|+|d u|^{2}$ from Theorem 4.1, we can 
apply Lemma 8.1 to find

$$
\begin{aligned}
|\Phi|_{0}^{2}\left(t, x_{0}\right) & \leq e^{c t}|\Phi|^{2}\left(t, x_{0}\right) \cdot \tilde{\eta}\left(t, x_{0}\right) \leq e^{c t} \sup _{x \in B_{R / 4}^{t}\left(x_{0}\right)}\left[|\Phi|_{0}^{2}(t, x) \cdot \tilde{\eta}(t, x)\right] \\
& \leq e^{c t} \cdot 2 \sup _{x \in B_{R}^{0}\left(x_{0}\right)}\left[|\Phi|_{0}^{2}(0, x) \cdot \tilde{\eta}(0, x)\right] \cdot e^{C \tilde{C} t / R^{2}} \\
& \leq 2 e^{c t} \sup _{x \in B_{R}^{0}\left(x_{0}\right)}\left[|\Phi|_{0}^{2}(0, x)\right] \cdot e^{C \tilde{C} t / R^{2}}
\end{aligned}
$$

for a constant $c$ depending only on $n, k_{0}, c_{0}$, where $c_{0}:=\sup _{\Sigma}|d \tilde{u}|_{0}^{2}$. Since $\bar{B}_{R}^{0}\left(x_{0}\right)$ is compact, the supremum is attained at some point $x^{*} \in B_{R}^{0}\left(x_{0}\right)$. Multiplying by $\tilde{r}^{6}\left(x_{0}\right)$ leads to

$$
|\Phi|_{0}^{2}\left(t, x_{0}\right) \cdot \tilde{r}^{6}\left(x_{0}\right) \leq 2 e^{c t} e^{C \tilde{C} t / R^{2}} \cdot|\Phi|_{0}^{2}\left(0, x^{*}\right) \cdot \tilde{r}^{6}\left(x_{0}\right) .
$$

Examining the constants, we see that either $\tilde{C}=1$ and $e^{C \tilde{C} t / R^{2}} \leq e^{C t / \rho^{2}}$ or $\tilde{C}=R^{2} \cdot \sqrt{K}$ such that $e^{C \tilde{C} t / R^{2}} \leq e^{C \sqrt{K} t}$. In both cases, we get $e^{c t} e^{C \tilde{C} t / R^{2}} \leq$ $e^{\bar{c} t}$ for a constant $\bar{c}=\bar{c}\left(n, k_{0}, c_{0}, s_{0}, \rho\right)$ independent of $x_{0}$, where $s_{0}:=\sup _{\Sigma}$ $\left|\tilde{\nabla}^{2} \tilde{u}\right|_{0}^{2}$. On all of $B_{R}^{0}\left(x_{0}\right)$, there is the estimate

$$
\tilde{r}(x) \geq \tilde{r}\left(x_{0}\right)-R=\frac{3}{4} \tilde{r}\left(x_{0}\right)
$$

such that we obtain

$$
|\Phi|_{0}^{2}\left(t, x_{0}\right) \cdot \tilde{r}^{6}\left(x_{0}\right) \leq 12 e^{\bar{c} t} \cdot\left(|\Phi|_{0}^{2}\left(0, x^{*}\right) \cdot \tilde{r}^{6}\left(x^{*}\right)\right) \leq 12 \bar{C}^{2} e^{\bar{c} t}
$$

Note that the choice of $R$ guarantees that the ball $B_{R}^{0}\left(x_{0}\right)$ is fully contained in $\Sigma_{K}$. Furthermore, the constants $\bar{c}$ and $\bar{C}$ do not depend on $x_{0}$. This implies that the estimate is uniform for all $x \in \Sigma \backslash B_{2 \rho}^{0}(O)$ and we have

$$
|\Phi|_{0}(t, x) \leq A \cdot \tilde{r}^{-3}(x)
$$

on $[0, T] \times \Sigma \backslash B_{2 \rho}^{0}(O)$ for a constant $A=A(t)$ depending only on $t$ with $A(t) \rightarrow \infty$ for $t \rightarrow \infty$. In the same way as above, we get using Lemma 8.2

$$
|d u|_{0}^{2}(t, x) \leq B \cdot \tilde{r}(x)^{-4}
$$


on $[0, T] \times \Sigma \backslash \bar{B}_{2 \rho}\left(x_{0}\right)$ with $B(t) \rightarrow \infty$ for $t \rightarrow \infty$. The estimates (8.3) and (8.4) allow us to integrate pointwise for all $x \in \Sigma \backslash B_{2 \rho}^{0}(O)$

$$
\begin{aligned}
\left|g(t)-\left(1-\frac{2 m}{\tilde{r}}\right) \delta\right|_{0} \leq & C_{0} \tilde{r}^{-2}+\int_{0}^{t}\left|\partial_{t} g(\tau)\right|_{0} d \tau \leq \tilde{C}_{0} \tilde{r}^{-2} \\
& +2 \alpha_{n} \int_{0}^{t}\left(|R c|_{0}+|d u|_{0}^{2}\right)(\tau) d \tau \\
\leq & \tilde{C}_{0} \tilde{r}^{-2}+\left(A \tilde{r}^{-3}+B \tilde{r}^{-4}\right) \cdot T=\tilde{C}_{0} \cdot \tilde{r}^{-2}
\end{aligned}
$$

where $\tilde{C}_{0}=\tilde{C}_{0}\left(n, C_{0}, A, B, T\right)$ is independent of $x$. For $u(t)$, we integrate

$$
\begin{aligned}
\left|u(t)+\frac{m}{r}\right|_{0} & \leq D_{0} \tilde{r}^{-2}+\int_{0}^{t}\left|\partial_{t} u(\tau)\right|_{0} d \tau \leq D_{0} \tilde{r}^{-2}+\int_{0}^{t}|\Delta u|_{0}(\tau) d \tau \\
& \leq D_{0} \tilde{r}^{-2}+n A T \cdot C \tilde{r}^{-3}=\tilde{D}_{0} \cdot \tilde{r}^{-2}
\end{aligned}
$$

where $\tilde{D}_{0}=\tilde{D}_{0}\left(n, D_{0}, A, B, T\right)$ also does not depend on $x$. This shows that $(g, u)(t)$ remains asymptotically flat at the zeroth order on $\Sigma \backslash B_{2 \rho}^{0}(O)$.

To estimate the first derivative of $g$, we need the first derivative of $\Phi$. From Lemma 5.8, we have

$$
\left(\partial_{t}-\Delta\right)|\nabla \Phi|^{2} \leq-2\left|\nabla^{2} \Phi\right|^{2}+C\left\{|\Phi||\nabla \Phi|^{2}+|d u||\Phi|^{2}|\nabla \Phi|+|d u|^{2}|\nabla \Phi|^{2}\right\} .
$$

Estimating $\left(|d u|^{2}+|\Phi|\right) \leq \tilde{C} R^{-2}$ as before and using Young's inequality, this implies

$$
\left(\partial_{t}-\Delta\right)|\nabla \Phi|^{2} \leq-2\left|\nabla^{2} \Phi\right|^{2}+C \tilde{C} R^{-2}|\nabla \Phi|^{2}+A^{4} \tilde{r}^{-12}
$$

The remaining calculations go through as above such that we find for $f:=$ $|\nabla \Phi|^{2} \cdot \tilde{\eta}:$

$$
\left(\partial_{t}-\Delta\right) f \leq C \tilde{C} R^{-2} f+C A^{4} \tilde{r}^{-12}\left(x_{0}\right) .
$$

Here we used that $\tilde{\eta} \leq 1$ on $B\left(T, x_{0}, R\right)$ and $\tilde{r}^{-1}(x) \leq \frac{4}{3} \tilde{r}^{-1}\left(x_{0}\right)$. Let $v(t)$ be the solution to

$$
\frac{d}{d t} v(t)=C \tilde{C} R^{-2} v(t)+C A^{4} \tilde{r}^{-12}\left(x_{0}\right)
$$

with initial value $v(0):=\frac{9}{8} \sup _{x \in B_{R}^{0}\left(x_{0}\right)} f(0, x)$. Then $v$ is given explicitly by

$$
\begin{aligned}
v(t) & =-C A^{4} \tilde{r}^{-12}\left(x_{0}\right) \cdot \frac{R^{2}}{C \tilde{C}}+e^{C \tilde{C} t / R^{2}} \cdot\left(v(0)+C A^{4} \tilde{r}^{-12}\left(x_{0}\right) \cdot \frac{R^{2}}{C \tilde{C}}\right) \\
& \leq e^{C \tilde{C} t / R^{2}} \cdot\left(v(0)+C A^{4} \tilde{r}^{-10}\left(x_{0}\right)\right)
\end{aligned}
$$


from the choice of $R$ and $\tilde{C} \geq 1$. Since $v$ is a barrier for $f$ for $t>0$, we find

$$
\begin{aligned}
& \sup _{x \in B_{R / 4}^{t}\left(x_{0}\right)} {\left[|\nabla \Phi|^{2}(t, x) \cdot \tilde{\eta}(t, x)\right] } \\
& \leq 2\left(\sup _{x \in B_{R}^{0}\left(x_{0}\right)}\left[|\nabla \Phi|_{0}^{2}(0, x) \tilde{\eta}(0, x)\right]+C A^{4} \tilde{r}^{-10}\left(x_{0}\right)\right) \cdot e^{C \tilde{C} t / R^{2}} .
\end{aligned}
$$

The asymptotic conditions for $\left|\partial^{3} g\right|$ and $\left|\partial^{3} u\right|$ imply that for $x \in \Sigma \backslash B_{\rho}^{0}(O)$ at $t=0$, we have

$$
|\nabla \Phi|_{0}(0, x) \leq \bar{C} \cdot \tilde{r}^{-4}
$$

independent of $x$. We find at a point $x^{*} \in B_{R}^{0}\left(x_{0}\right)$ where the supremum is attained that

$$
\begin{aligned}
|\nabla \Phi|^{2}\left(t, x_{0}\right) \cdot \tilde{r}^{8}\left(x_{0}\right) & \leq\left(2|\nabla \Phi|_{0}^{2}\left(0, x^{*}\right) \cdot \tilde{r}^{8}\left(x_{0}\right)+C A^{4} \tilde{r}^{-10} \cdot \tilde{r}^{8}\left(x_{0}\right)\right) \cdot e^{C \tilde{C} t / R^{2}} \\
& \leq\left(32 \bar{C}+C A^{4} \tilde{r}^{-2}\left(x_{0}\right)\right) e^{C \tilde{C} t / R^{2}}=: E(t)
\end{aligned}
$$

holds, where $E(t)$ does not depend on $x$. Here we also used $\tilde{r}^{-1}\left(x_{0}\right) \leq 1$. This implies the decay

$$
|\nabla \Phi|_{0}\left(t, x_{0}\right) \leq E(t) \cdot \tilde{r}^{-4}\left(x_{0}\right)
$$

on $[0, T] \times B_{2 \rho}\left(x_{0}\right)$. Observe that $E(t) \rightarrow \infty$ for $t \rightarrow \infty$. Integration gives

$$
\begin{aligned}
|\partial g|_{0}(t) \leq & |\partial \tilde{g}|_{0}+\int_{0}^{t}\left|\partial_{t} \partial g\right|_{0}(\tau) d \tau \leq C_{1} \cdot \tilde{r}^{-3} \\
& +2 \alpha_{n} \sup _{\tau \in[0, T]}\left(|\nabla R c|_{0}+|d u|_{0}\left|\nabla^{2} u\right|_{0}\right)(\tau) \cdot T \\
\leq & C_{1} \cdot \tilde{r}^{-3}+2 \alpha_{n} T \cdot E \tilde{r}^{-4}+2 \alpha_{n} T \sqrt{B} A \tilde{r}^{-5} \leq \tilde{C}_{1} \cdot \tilde{r}^{-3} .
\end{aligned}
$$

Together with (8.4), we get the desired asymptotics for $\partial g$ and $\partial u$. We have from (8.5) and (8.6)

$$
\begin{aligned}
& \left|\partial^{2} g\right|_{0} \leq|R m|_{0}+|\partial g|_{0}^{2} \leq A \cdot \tilde{r}^{-3}+\tilde{C}_{1}^{2} \cdot \tilde{r}^{-4} \leq \tilde{C}_{2} \cdot \tilde{r}^{-3}, \\
& \left|\partial^{2} u\right|_{0} \leq\left|\nabla^{2} u\right|_{0}+|\partial g|_{0}|\partial u|_{0} \leq A \cdot \tilde{r}^{-3}+\tilde{C}_{1} \cdot \tilde{r}^{-2} \cdot \tilde{D}_{1} \cdot \tilde{r}^{-2} \leq D_{2} \cdot \tilde{r}^{-3},
\end{aligned}
$$

establishing the claim for $k=2$. The remaining case $k=3$ is handled analogously. Since $\tilde{g}$ and $\delta$ are equivalent, this proves that the asymptotic flatness is preserved. The estimates (8.5) and (8.6) show that the change of $(g, u)$ in time is of order $\tilde{r}^{-2}$. Therefore the asymptotic form (8.1) and (8.2) of the initial data is preserved. It immediately follows that the ADM mass stays constant under the flow (3.1). 
The same can be proved for the following weaker definition of asymptotic flatness.

Definition 8.5. Let $\Sigma$ be a complete $n$-dimensional connected Riemannian manifold. $(\Sigma, g, u)$ is called asymptotically flat, if there is a compact subset $K \subset \Sigma$ such that $\Sigma_{K}:=\Sigma \backslash K$ is diffeomorphic to $\mathbb{R}^{n} \backslash B_{1}(0)$ and $(g, u)$ satisfy in the exterior region $\Sigma_{K}$

$$
\begin{aligned}
|g-\delta|+|u| & \leq C_{0} \cdot \tilde{r}^{-1}, \\
\left|\partial^{k} g\right|+\left|\partial^{k} u\right| & \leq C_{k} \cdot \tilde{r}^{-k-1}, \quad k=1,2,3,
\end{aligned}
$$

for constants $C_{k}$, where $\tilde{r}(x):=\sqrt{x_{1}^{2}+\cdots+x_{n}^{2}}$ is the radial coordinate of $\mathbb{R}^{n}$ and $|\cdot|$ and $\partial$ are with respect to the Euclidean metric.

Theorem 8.6. Let $(g, u)(t)$ be the solution to $(3.1)$ on $[0, T] \times \Sigma$ for $T<$ $\infty$ with initial data $(\tilde{g}, \tilde{u})$ given by Theorem 4.1. Assume that $(\Sigma, \tilde{g}, \tilde{u})$ is asymptotically flat with $K=\bar{B}_{\rho}^{0}(O)$ for constants $C_{k}$. Then $(\Sigma, g(t), u(t))$ is asymptotically flat for all $t \in[0, T]$. In particular, there is a constant $C(t)=$ $C\left(t, k_{0}, c_{0}, s_{0}, C_{k}, n\right)$ depending only on time, the bounds from Theorem 4.1 and the asymptotic decay of $(\tilde{g}, \tilde{u})$ such that

$$
\begin{aligned}
|g(t)-\delta|_{0}+|u(t)|_{0} & \leq C(t) \cdot \tilde{r}^{-1} \\
\left|\partial^{k} g\right|_{0}+\left|\partial^{k} u\right|_{0} & \leq C(t) \cdot \tilde{r}^{-k-1}
\end{aligned}
$$

holds on $[0, T] \times \Sigma \backslash B_{2 \rho}(O)$ for $k=1,2,3$, where $C(t) \rightarrow \infty$ for $t \rightarrow \infty$. In addition, the ADM mass stays constant in time and we have $m_{A D M}(g(t))=$ $m_{A D M}(\tilde{g})$ for all $t \in[0, T]$.

Proof. The proof is analogous to the proof of Theorem 8.4. The integration (8.5) of the metric does not change the first-order term in $\tilde{r}$ which determines the mass of $g(t)$.

Remark 8.7. Although the mass is constant for solutions of (3.1) on finite time intervals, we expect that the mass $m_{\mathrm{ADM}}\left(g_{\infty}\right)$ of the limit $\left(g_{\infty}, u_{\infty}\right)$ of a global solution $(g, u)(t)$ for $t \rightarrow \infty$ jumps. In particular, it is not clear that the limit in time commutes with the spatial limit.

\section{Acknowledgements}

The author would like to express his gratitude to Prof. Dr. Gerhard Huisken for his advice and encouragement while this thesis was carried out and to the Max Planck Gesellschaft for the research opportunity and financial support. 


\section{References}

[1] R.S. Hamilton, Three-manifolds with positive Ricci curvature, J. Differ. Geom. 17 (1982), 255-306.

[2] - The formation of singularities in the Ricci flow, Surveys in Differential Geometry, 2, International Press Inc., Boston, 1995.

[3] - Four-manifolds with positive curvature operator, J. Differ. Geom. 24 (1986), 153-179.

[4] - A compactness property for solutions of the Ricci flow, Amer. J. Math. 117 (1995), 545-572.

[5] - Four-manifolds with positive isotropic curvature, Comm. Anal. Geom. 5(1) (1997), 1-92.

[6] G. Perelman, The entropy formula for the Ricci flow and its geometric applications, 2002. http://arxiv.org/abs/math.DG/0211159.

[7] - Ricci flow with surgery on three-manifolds, 2003. http://arxiv.org/abs/math.DG/0303109.

[8] A. Rendall, Theorems on existence and global dynamics for the Einstein equations, Living Rev. Relat. 8(6) (2005). URL (cited on 12/12/05): http://www.livingreviews.org/lrr-2005-6.

[9] G. Cook, Initial data for numerical relativity, Living Rev. Relativ. 3(5) (2000). URL (cited on 12/12/05): http://www.livingreviews.org/lrr2000-5.

[10] R. Bartnik, Energy in general relativity, Tsing Hua Lectures on Geometry \& Analysis (Hsinchu, 1990-1991), 1997, 5-27.

[11] P. Miao, On existence of static metric extensions in general relativity, 2003. http://arxiv.org/abs/math-ph/0309041v1.

[12] J. Ehlers and W. Kundt, Exact solutions of the gravitational field equations, in Gravitation: an Introduction to Current Research, ed. L. Witten, John Wiley \& Sons, Inc., New York, London, 1962, 49-101.

[13] R. Wald, General Relativity, The University of Chicago Press, Chicago, London, 1984.

[14] R. Beig and B. Schmidt, Time-independent gravitational fields, in Einstein's Field Equations and Their Physical Implications, ed. B. Schmidt, Springer Verlag, Berlin, Heidelberg, 1999, 325-372. 
[15] B. List, Evolution of an extended Ricci flow system, PhD thesis, 2006. http://www.diss.fu-berlin.de/2006/180/index.html.

[16] D. DeTurck, Deforming metrics in the direction of their Ricci tensors, J. Differ. Geom. 18 (1983), 157-162.

[17] W.-X. Shi, Deforming the metric on complete Riemannian manifolds, J. Differ. Geom. 30 (1989), 223-301.

[18] B. Chen and X. Zhu, Uniqueness of the Ricci flow on complete noncompact manifolds, 2005. http://arxiv.org/abs/math.DG/0505447v3.

[19] T. Oliynyk, Renormalization group flow on asymptotically flat manifolds, 2005, Preprint.

[20] R. Schoen and S.T. Yau, Lectures on differential geometry, Conference Proceedings and Lecture Notes in Geometry and Topology, 1, International Press Inc., Boston, 1994.

[21] K. Ecker and G. Huisken, Interior estimates for hypersurfaces moving by mean curvature, Invent. Math. 105(3) (1991), 547-569.

[22] B. Chow and D. Knopf, The Ricci flow: an introduction, volume Mathematical Surveys and Monographs, 110, American Mathematical Society, Providence, RI, 2004.

[23] O.S. Rothaus, Logarithmic Sobolev inequalities and the spectrum of Schrödinger operators, J. Funct. Anal. 42 (1981), 110-120.

[24] J. Cheeger, M. Gromov and M. Taylor, Finite propagation speed, kernel estimates for functions of the Laplace operator, and the geometry of complete Riemannian manifolds, J. Differ. Geom. 17 (1982), 15-53.

[25] R. Bartnik, The mass of an asymptotically flat manifold, Comm. Pure Appl. Math. 39(5) (1986), 661-693.

MaX-Planck-Institut Für Gravitationsphysik

Albert-Einstein-Institut

AM MÜHLENBERG 1

D-14467 PotSDAM

GERMANY

E-mail address: bernhard.1ist@web.de

Received November 20, 2006 